\title{
Randomized Spectral Separation Coefficient for Short Code Acquisition Performance Evaluation
}

\author{
Christoph Enneking, Felix Antreich, Senior Member, IEEE, \\ and André L. F. de Almeida, Senior Member, IEEE
}

\begin{abstract}
Reliable signal acquisition with low computational complexity is an important design objective for the evolution of global navigation satellite systems (GNSS). Most GNSS signals consist of long pseudorandom noise (PRN) codes whose acquisition is expensive in terms of memory, computation time, and energy. As these resources are particularly scarce in the emerging mass-market user segment, cyclostationary pilot signals with short PRN codes are an attractive option to keep the number of acquisition search bins low. However, reducing the code length degrades the acquisition performance, as multiple access interference (MAI) becomes more pronounced and can lead to an increased false alarm rate. We demonstrate that, quite different from stationary MAI, cyclostationary MAI does not affect each bin of the search space uniformly, and is therefore not easily modeled with the well-known spectral separation coefficient (SSC). We propose a new randomized SSC (SSC-R) based on code/Doppler interference functions, which can be used for simple and accurate acquisition performance evaluation. As an application example, we demonstrate how the SSC-R can be utilized in signal design to minimize the PRN code length under an acquisition performance constraint. We conclude that feasible PRN code lengths for GNSS can be on the order of 300-700.
\end{abstract}

Index Terms-Global navigation satellite system (GNSS), global positioning system (GPS), coarse/acquisition (C/A), selfinterference.

\section{INTRODUCTION}

$\mathbf{S}$ IGNAL acquisition is a resource-hungry process for receivers of global navigation satellite systems (GNSS). A GNSS satellite transmits pseudorandom noise (PRN) code and navigation data which arrive at the receiver with low signalto-noise ratio, unknown code-phase, and unknown Doppler frequency. To acquire such a signal, the receiver must correctly detect that the signal is actually present at the receive antenna, and estimate the two unknown synchronization parameters code-phase/Doppler with coarse resolution [1]. The necessary 2-D search for correlation over a set of code-phase/Doppler hypotheses (bins) requires considerable computation time (if

Manuscript submitted December, 2020. This work has been carried out within the framework of the project "R\&D for maritime safety and security and corresponding real time services" led by the Program Coordination Defence and Security Research within the German Aerospace Center (DLR). This work was also supported in part by CNPq - Brazilian National Research Council - under grant 309248/2018-3 PQ-2.

(Corresponding author: C. Enneking.)

C. Enneking is with the German Aerospace Center (DLR), Oberpfaffenhofen, Germany (e-mail: christoph.enneking@dlr.de). A. L. F. de Almeida and C. Enneking are with the Federal University of Ceará (UFC), Fortaleza, Brazil (e-mail: andre@gtel.ufc.br). F. Antreich is with the Aeronautics Institute of Technology (ITA), São José dos Campos, Brazil (e-mail: antreich@ieee.org). bins are searched sequentially), memory (if bins are searched in parallel), and in any case energy [2]-[5]. Besides these complexity measures, the global probability of false alarm (GPF) and the global probability of detection (GPD) indicate the statistical reliability of the acquisition in the presence of nuisances such as noise, interference, or navigation data transitions [6], [7]. Together, GPF and GPD form the receiver operating characteristic (ROC). In case of a false alarm or missed detection, even more receiver resources are consumed, e.g. due to false initialization of tracking loops or due to an acquisition restart.

The computational expense of GNSS signal acquisition poses a major challenge especially to mass-market consumer electronics such as mobile phones, asset trackers, or internet of things devices [4], [5], [8]. Typically, such receivers are only occasionally prompted to provide positioning or navigation, so that they spend much of their duty cycle in acquisition mode. Moreover, they are built on very small integrated circuits with limited memory, but are expected to acquire several signals within few seconds and to economize the precious battery energy. A compromise between memory and computation time can be achieved, using serial, parallel, or hybrid search techniques [9], [10]. The consumed energy, however, is essentially a constant proportional to the number of bins and cannot be traded off at the cost of some other resource. Despite the widespread use of assisted GNSS [5], [11], which can help to exclude some regions of the search space $a$ priori, the overall number of bins to be searched is still considerable, especially if the signal's PRN code is long.

(Quasi-)Pilot GNSS signals have been designed [12]-[15] or re-designed [16] to address emerging mass-market user needs. So far, these designs have focused on the use of very low data rates $(0-50 \mathrm{~Hz})$ to avoid sensitivity loss due to data symbol transitions, i.e., they enhance the GPD. The successful GPS L1 C/A signal (50 Hz data / 1023 chips PRN code) has served as a blueprint in most of these considerations. Interestingly, another apparently obvious signal design option has not yet been evaluated systematically, and that is the reduction of the PRN code length. The decisive feature which makes L1 C/A an extremely attractive acquisition signal is not its low data rate, but the combination of a low data rate with a relatively short PRN code, as is widely agreed [5], [11], [16]. This feature leads to a low number of search bins and a decent ROC performance, and is currently offered by no other GNSS service, which gives L1 C/A a competitive edge in consumer 
GNSS to the present day. As the authors of [16] point out, this feature (more precisely, the repetition of more than one PRN code period during the transmission of a single data symbol) has a potentially hazardous implication though. Signals with this feature cease to be stationary and are especially vulnerable to multiple access interference (MAI) caused by other satellites transmitting the same service. It is known that MAI can create a detectable false correlation peak in near-far scenarios [17]. Under nominal received power levels, it needs to be ensured qua design that the ROC is not degraded excessively by MAI.

The theory of spectral separation coefficients (SSCs) is a powerful tool to model the MAI between any two signals, in particular, MAI between a locally generated PRN code of interest and another interfering PRN. While the standard SSC as originally proposed by Betz [18], [19] is only accurate for stationary signals, more accurate versions of the SSC have been proposed which also work well with GPS L1 C/A and similar cyclostationary signals [20]-[24]. While these proposed modifications of the SSC vary slightly in form or value, they agree in that the SSC shoud be modeled as a function of the involved signals' Doppler frequencies, and possibly even more channel parameters such as (fractional) code-phase. Therefore, they all have in common that they depend on channel parameters and treat these as deterministic, which is why we summarize these variants under the term deterministic SSC (SSC-D). Computed over an acquisition search grid, the SSC-D would depend on more than a few parameters, and can therefore not readily be applied to model the ROC. Such a straightforward application of the SSC-D would simply require considerable processing, as the MAI from a set of interfering satellites on each local replica of the 2-D search space would have to be evaluated explicitly. Doing this during a system optimization process, in which the PRN code length is a design parameter, would be computationally prohibitive.

In this work, we propose a methodology to assess the impact of cyclostationary MAI on the ROC for any given PRN code length. Our approach is based on the SSC-D, but a randomized version thereof called SSC-R. The SSC-R approximates the SSC-D as a random variable (RV). A key assumption is that MAI can be approximated as independent and identically distributed (i.i.d.) RVs over all bins. This approximation obviates the need to compute the SSC-D explicitly for each bin, but allows to work with one representative probability density function (PDF) instead. Our approach is a trade-off between the accuracy of the SSC-D and the simplicity of the SSC, simple enough to use for optimization of system parameters. As an application example, we demonstrate how the proposed methodology can be used to minimize the PRN code length, while ensuring constraints on reliability and sensitivity. For simplicity, we only consider intrasystem MAI in this work (all users have the same symbol rate and code length), but the approach can be easily generalized to intersystem MAI using [22].

The rest of this paper is organized as follows. In Section II, we define the acquisition system, consisting of the received signal model, the generation of decision statistics, and the search strategy. In Section III, we review SSC and SSC-D, develop the novel SSC-R, and derive models for the probability of false alarm under either SSC version. In Section IV, we model the probability of detection, taking into account MAI, symbol transitions, and finite search resolution. In Section V, the validity of the SSC-R is demonstrated at the example of numerical simulations. Finally, we present an application of the proposed model for signal design in Section VI.

\section{System Model}

We consider $K$ satellite signals received over an additive white Gaussian noise (AWGN) channel, where one signal is to be acquired and the remaining $K-1$ signals are interference. For $P$ code bins and $Q$ Doppler bins, we describe the processing steps for the generation of $P Q$ decision statistics based on the same set of input baseband samples. An arbitrary but fixed number of coherent and noncoherent summations is considered. Subsequently, we briefly describe the search strategy for serial and parallel search.

\section{A. Baseband Signal}

The received signal in complex baseband notation is

$$
r(t)=\sum_{k=1}^{K} \sqrt{P_{k}} x_{k}(t)+w(t),
$$

where the $k$ th satellite contributes the signal $x_{k}(t)$ with power $P_{k}$, while the real and imaginary part of $w(t)$ are two independent white Gaussian noise processes each with twosided PSD $N_{0} / 2$. A model for $x_{k}(t)$ is shown at the top of Fig. 2. For $k=1, \ldots, K$, the satellite signals are given as

$$
x_{k}(t)=e^{\mathrm{j} 2 \pi \nu_{k} t+\mathrm{j} \varphi_{k}} \sum_{m=-\infty}^{\infty} s_{k}\left(t-m T_{0}-\tau_{k}\right) b_{k}[m] .
$$

The parameters $\tau_{k}, \nu_{k}$, and $\varphi_{k}$ denote to the unknown codephase, Doppler frequency, and carrier-phase, respectively. The signal $s_{k}(t)$ is a known code waveform, which repeats with code rate $1 / T_{0}$ and is modulated by a symbol sequence $b_{k}[m] \in\{-1,+1\}$. We consider only direct-sequence codedivision multiple access waveforms of the structure

$$
s_{k}(t)=\sum_{j=0}^{N_{c}-1} c_{k}[j] h\left(t-j T_{c}\right),
$$

where $c_{k}[j]$ is a known PRN code, and $h(t)$ is a realvalued analog pulse shape (for instance, a rectangular pulse). Therefore, the code period is equal to $T_{0}=N_{c} T_{c}$, where $N_{c}$ is the code length and $T_{c}$ is the pulse duration. For $k=1, \ldots, K$, the code-phase, carrier-phase, and Doppler frequency are assumed to be independent RVs on the intervals $\left(-T_{0} / 2, T_{0} / 2\right),(-\pi, \pi)$, and $\left(-F_{0} / 2, F_{0} / 2\right)$, respectively, with some Doppler span $F_{0} \geqslant 0$.

We introduce the pulse's Fourier transform $H(f) \triangleq$ $\int_{\mathbb{R}} h(t) e^{-\mathrm{j} 2 \pi f t} \mathrm{~d} t$ and its autocorrelation function $\rho_{h}(t) \triangleq$ $\int_{\mathbb{R}} h(\tau) h(\tau+t) \mathrm{d} \tau$, with normalization $\rho_{h}(0)=T_{c}$. For simplicity, we assume that the pulse satisfies the Nyquist condition $\rho_{h}\left(n T_{c}\right)=0$ for all $n= \pm 1, \pm 2, \ldots$ This condition is fulfilled by many pulse shapes that are relevant for GNSS, 


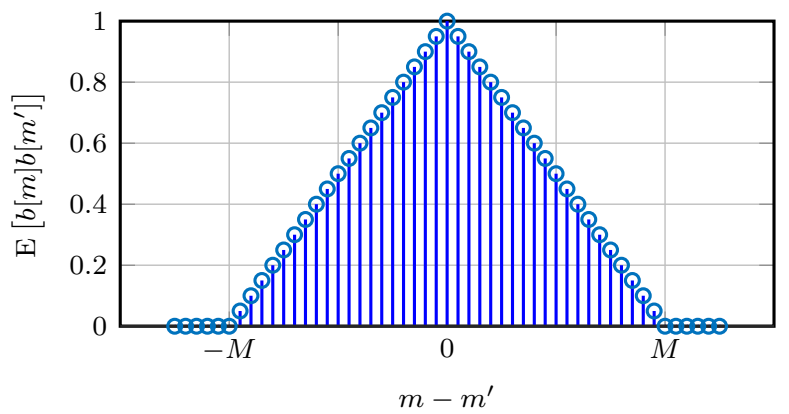

Fig. 1. Autocorrelation of the binary symbol sequence $b_{k}[m]$, with symbol boundaries every $M$ elements. In this example, $M=20$ (e.g. GPS L1 C/A).
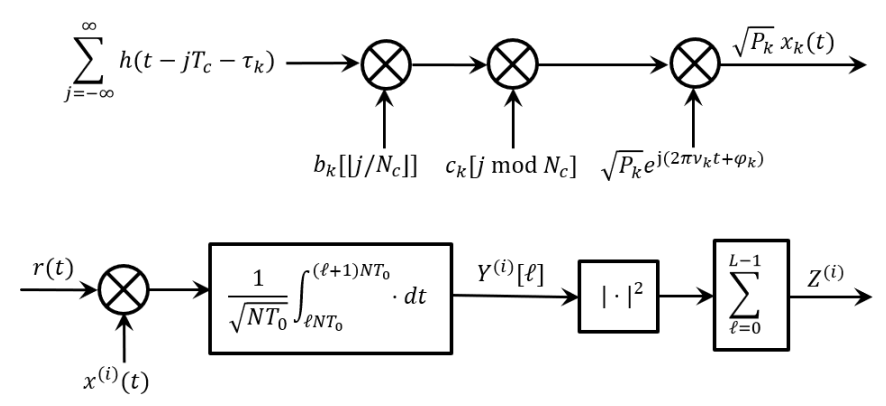

Fig. 2. Top: $k$ th transmitter. Bottom: $i$ th bin decision statistic.

e.g. by all pulses which are zero outside the interval $\left(-T_{c}, T_{c}\right)$, or by the root-raised cosine (RRC) pulse.

The statistical properties of the PRN code $c_{k}[j]$ and the symbol sequence $b_{k}[\mathrm{~m}]$ are an important aspect of our analyses, as they can have a fundamental impact on the distribution of MAI. We model the PRN code $c_{k}[0], \ldots, c_{k}\left[N_{c}-1\right]$ as a coin-flip sequence of length $N_{c}$ (i.e., $N_{c}$ i.i.d. binary RVs which assume values $\{-1,+1\}$ with equal probability). The values of the symbol sequence $b_{k}[m]$ are equiprobable in $\{-1,+1\}$ and are not, in general, independent, as transitions can only occur every $M$ elements. Mathematically, this can be expressed as $b_{k}[m]=d_{k}\left[\left\lceil\left(m-\vartheta_{k}\right) / M\right\rceil\right]$, where $d_{k}[m]$ is an infinite coin-flip sequence, $\vartheta_{k} \in\{1, \ldots, M\}$ is a uniformly random initial symbol-phase, and $M \in \mathbb{N}$. The autocorrelation of such a symbol sequence (for random $\vartheta_{k}$ ) is a triangular sequence [20], [24] as shown in Fig. 1. The PRN code $c_{k}[j]$ and the symbol sequence $b_{k}[m]$ are assumed mutually independent, and also independent for $k=1, \ldots, K$. Note that we model both as truly random, although in fact PRN code will always and symbols (e.g. secondary code) may sometimes be pseudorandom.

It is important to distinguish between the symbol rate $1 /\left(M T_{0}\right)$ and the code rate $1 / T_{0}$. A few typical setups are worth mentioning: (i) balanced: $M=1$; (ii) quasi-pilot: $M \gg 1$; (iii) pure pilot: $M \rightarrow \infty$. The balanced configuration (i) is typical for modernized civil GNSS services such as the Galileo Open Service [25], and will reduce the autocorrelation in Fig. 1 to a unit impulse. In this work, we will focus on (ii) and (iii), as they are the most attractive options for acquisition signals, and the most prone to MAI.

\section{B. Decision Statistics}

Without loss of generality, we consider $k=1$ as the signal of interest (SOI). The acquisition task is specified as follows. Decide for either of the following hypotheses:

- $H_{0}$ : the SOI is absent $\left(P_{1}=0\right)$;

- $H_{1}$ : the SOI is present $\left(P_{1}>0\right)$;

additionally, if the decision is taken for $H_{1}$, select a coarse estimate for $\tau_{1}$ from a set of code-phase candidates $\mathcal{X}_{\tau}$, and a coarse estimate for $\nu_{1}$ from a set of Doppler candidates $\mathcal{X}_{\nu}$.

These candidate sets form a 2-D grid of bins with $P$ codephases and $Q$ Doppler frequencies, distributed uniformly over the uncertainty intervals $\left(-T_{0} / 2, T_{0} / 2\right)$ and $\left(-F_{0} / 2, F_{0} / 2\right)$, respectively. Thus we have

$$
\begin{aligned}
& \mathcal{X}_{\tau}=\left\{-\frac{T_{0}+\Delta \tau}{2},-\frac{T_{0}+3 \Delta \tau}{2}, \ldots, \frac{T_{0}-\Delta \tau}{2}\right\}, \\
& \mathcal{X}_{\nu}=\left\{\frac{-F_{0}+\Delta \nu}{2}, \frac{-F_{0}+3 \Delta \nu}{2}, \ldots, \frac{F_{0}-\Delta \nu}{2}\right\},
\end{aligned}
$$

with code-phase spacing $\Delta \tau=T_{0} / P$ and frequency spacing $\Delta \nu=F_{0} / Q$. We use a linear index $i \in\{1, \ldots, P Q\}$ to refer to the 2-D bin $\left(\tau^{(i)}, \nu^{(i)}\right) \in\left(\mathcal{X}_{\tau} \times \mathcal{X}_{\nu}\right)$. We define the correct bin with index $i=1$ as the bin that deviates the least from the true parameters in the sense that

$$
\left|\tau^{(1)}-\tau_{1}\right| \leqslant \Delta \tau / 2 \text { and }\left|\nu^{(1)}-\nu_{1}\right| \leqslant \Delta \nu / 2
$$

It is easily checked that this assignment is unique, i.e., there is always one correct bin with probability one. ${ }^{1}$ The remaining bins $i=2, \ldots, P Q$ are in no particular order yet.

For each bin $i$, an associated statistic $Z^{(i)}$ is generated as follows. First, the receiver performs the correlation of the received signal $r(t)$ with the $i$ th local replica

$$
x^{(i)}(t) \triangleq e^{\mathrm{j} 2 \pi \nu^{(i)}} t \sum_{n=-\infty}^{\infty} s_{1}\left(t-n T_{0}-\tau^{(i)}\right),
$$

The structure of $x^{(i)}(t)$ is the same as of the SOI $x_{1}(t)$, except from the modulating symbol sequence, which is assumed unknown to the receiver. Correlation with the local replica can thus also be viewed as matched filtering with respect to pulse shape and PRN code. Correlating during the coherent integration time $T$ leads to the correlator output

$$
Y^{(i)}[\ell]=\frac{1}{\sqrt{T}} \int_{\ell T}^{(\ell+1) T} \overline{x^{(i)}(t)} r(t) \mathrm{d} t
$$

for coherent subintervals $\ell=0, \ldots, L-1$. We consider only integration times which are an integer multiple of the code period, i.e., $T=N T_{0}$. Secondly, the $L$ correlator outputs are noncoherently combined

$$
Z^{(i)}=\sum_{\ell=0}^{L-1}\left|Y^{(i)}[\ell]\right|^{2} .
$$

The total dwell time for the generation of $Z^{(i)}$ is $L T$. One possible receiver implementation is described at the bottom of Fig. 2.

\footnotetext{
${ }^{1}$ Special cases, where the true code-phase and/or Doppler frequency lie exactly at a bin boundary, have probability measure zero.
} 


\section{Decision criterion}

We consider only acquisition strategies based on threshold comparison, implemented either as serial or parallel search.

1) Serial search: Determine the starting index $j \in$ $\{1, \ldots, P Q\}$ (uniformly random or according to prior knowledge). Serially for $i=j, j-1, \ldots, 1, P Q, P Q-1, \ldots, j+1$, compare the statistic $Z^{(i)}$ to a fixed threshold $\lambda \geqslant 0$. As soon as $Z^{(i)}>\lambda$, immediately accept $H_{1}$, return the bin $i$, and terminate the search. ${ }^{2}$ If no statistic has exceeded the threshold after $P Q$ comparisons, accept $H_{0}$.

2) Parallel search: Compare all statistics to the threshold in parallel. Determine the subset $\mathcal{J}$ of all bins $j \in\{1, \ldots, P Q\}$ for which $Z^{(j)}>\lambda$. If $\mathcal{J}$ is non-empty, accept $H_{1}$ and return the bin $i=\arg \max _{j \in \mathcal{J}} Z^{(j)}$ as code-phase/Doppler estimate. Otherwise, accept $H_{0}$.

\section{Probability of False Alarm}

Let $H_{0}$ be the true hypothesis. We say that a bin false alarm occurs in the $i$ th bin if $Z^{(i)}>\lambda$ for any $i \in\{1, \ldots, P Q\}$ (regardless of whether the actual search is terminated before reaching the $i$ th bin). Moreover, a global false alarm is raised if at least one bin false alarm occurs (in that case, the receiver will erroneously decide for $H_{1}$ ). Let the unknown probabilities of these events be denoted by

- the bin probability of false alarm $(\mathrm{BPF}) P_{f}^{(i)}(\lambda)$;

- the global probability of false alarm (GPF) $P_{F}(\lambda)$.

Clearly, these probabilities do not depend on whether serial or parallel search is used [26].

In the following Sections III-A to III-D, we discuss four different models for the approximation of BPF and GPF, starting from a very simplistic AWGN-only model, moving on to the state-of-the-art models based on SSC and SSC-D, and finally presenting the novel model based on randomized SSCs.

All four models will make use of the following parameterized cumulative distribution function (CDF). Let the RV $Z$ be the sum of the squares of $L$ i.i.d. circularly-symmetric complex Gaussian (CSCG) RVs with mean zero and variance $\mathcal{N}_{0}$. Then $Z$ has the $\mathrm{CDF}$

$$
F_{Z}\left(z ; \mathcal{N}_{0}\right) \triangleq 1-e^{-\frac{z}{\mathcal{N}_{0}}} \sum_{\ell=0}^{L-1} \frac{1}{\ell !}\left(\frac{z}{\mathcal{N}_{0}}\right)^{\ell}, z \geqslant 0
$$

This is a scaled version of the CDF of a central $\chi^{2}$-distribution with $2 L$ degrees of freedom.

\section{A. AWGN Performance}

We begin with the BPF and GPF for the case of AWGN only, neglecting MAI. In the absence of MAI, it can be shown that $Y^{(i)}[1], \ldots, Y^{(i)}[L]$ are i.i.d. CSCG with mean zero and

$$
\operatorname{Var}\left[Y^{(i)}[\ell]\right]=N_{0}
$$

\footnotetext{
${ }^{2}$ Note that the search bins, for which we had defined no particular order yet, are simply indexed according to the serial search index sequence.
}

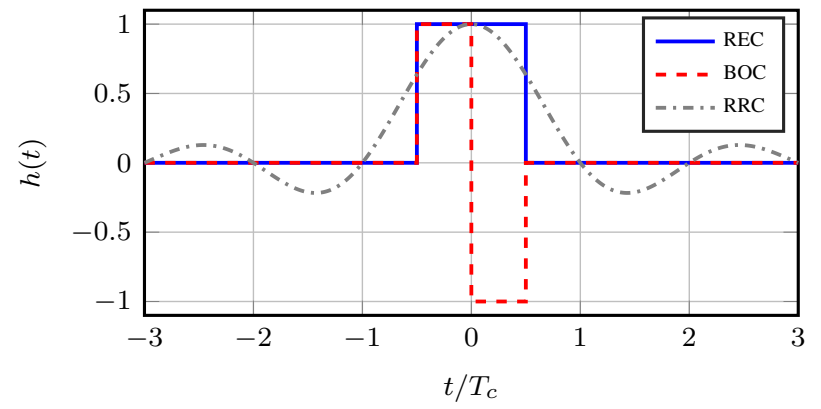

Fig. 3. Pulse shapes: rectangular (REC), binary offset carrier (BOC), rootraised cosine (RRC) with zero roll-off factor.

A short proof for (11) is given in the Appendix. Now the probability that $Z^{(i)}>\lambda$ can be expressed in terms of the parameterized CDF (10) as

$$
P_{f}^{(i)}(\lambda)=1-F_{Z}\left(\lambda ; N_{0}\right)
$$

and the GPF is simply

$$
P_{F}(\lambda)=1-\left(F_{Z}\left(\lambda ; N_{0}\right)\right)^{P Q} .
$$

\section{B. Standard SSC}

If MAI is present in addition to AWGN, this results in an increased correlator output variance

$$
\operatorname{Var}\left[Y^{(i)}[\ell]\right]=N_{0}+I_{0}
$$

where the contribution of MAI is

$$
\begin{aligned}
I_{0} & =\sum_{k=2}^{K} I_{k} \\
I_{k} & =P_{k} \mathrm{SSC} .
\end{aligned}
$$

The well-known SSC in units of $1 / \mathrm{Hz}$ is given by [18]

$$
\mathrm{SSC}=\frac{\int_{-\infty}^{\infty}|H(f)|^{4} \mathrm{~d} f}{\int_{-\infty}^{\infty}|H(f)|^{2} \mathrm{~d} f \int_{-\infty}^{\infty}|H(f)|^{2} \mathrm{~d} f}=T_{c} \alpha_{0}
$$

where we also defined the dimensionless coefficient $\alpha_{0} \triangleq$ $\frac{1}{T_{c}^{3}} \int_{-\infty}^{\infty}|H(f)|^{4} \mathrm{~d} f$ for reasons that will become evident later. $A^{c}$ proof for (14)-(17) can be found in the Appendix.

The SSC does not depend on the bin index $i$ or any channel parameters such as code-phase or Doppler frequency, but is determined solely by the pulse shape. As we consider MAI within a single service, interfering and desired signal both use the same pulse shape, so that the notion of spectral separation is somewhat misleading at this point. Therefore, the SSC in (17) is also known as self-SSC. Some common pulse shapes are shown in Fig. 3, and their self-SSCs are given in Table I.

To obtain BPF and GPF, we simply reuse results from the pure AWGN channel, replacing $N_{0}$ by $N_{0}+I_{0}$, to obtain

$$
\begin{aligned}
P_{f}^{(i)}(\lambda) & =1-F_{Z}\left(\lambda ; N_{0}+I_{0}\right) \\
P_{F}(\lambda) & =1-\left(F_{Z}\left(\lambda ; N_{0}+I_{0}\right)\right)^{P Q} .
\end{aligned}
$$


Table I

COMMON PULSE SHAPES: SSC AND INTERFERENCE FUNCTION FOURIER COEFFICIENTS

\begin{tabular}{c||c|ccc}
$h(t)$ & SSC & $\alpha_{0}$ & $\alpha_{1}$ & $\alpha_{2}$ \\
\hline REC & $2 T_{c} / 3$ & $2 / 3$ & $1 / \pi^{2}$ & $1 /\left(4 \pi^{2}\right)$ \\
BOC & $T_{c} / 3$ & $1 / 3$ & $1 / \pi^{2}$ & $5 /\left(4 \pi^{2}\right)$ \\
RRC & $T_{c}$ & 1 & 0 & 0
\end{tabular}

\section{Deterministic SSC (SSC-D)}

While the standard SSC model is very convenient, it is based on the assumption that the correlator output in the presence of MAI is still zero-mean CSCG distributed, hence fully characterized by its variance (14). Works in the context of satellite navigation have found that this is often not true, because the variance can increase or decrease considerably when it is computed conditioned on the signals' Doppler frequencies [20]-[24]. Moreover, research in terrestrial communications indicates that a comparable (albeit more subtle) effect can be observed when conditioning on the (fractional) code-phase [27], [28]. While this conditioning leads to a dependency of the variance on several channel parameters, it also improves the quality of the CSCG approximation of the correlator output, as was shown analytically by Zang and Ling [28], and later verified for satellite navigation scenarios [29].

These state-of-the-art findings are summarized in the following proposition. While these are known results and have been published in similar form across the above mentioned works, the detailed derivation of the combined effect of code-phase and Doppler frequency is unique to this paper. The proposition will also serve as a solid theoretical basis for the development of the SSC-R model.

Proposition 1. Let the interferers' random Doppler frequencies and code-phases be contained in the vectors $\nu \triangleq$ $\left[\nu_{2}, \ldots, \nu_{K}\right]^{T}$ and $\tau \triangleq\left[\tau_{2}, \ldots, \tau_{K}\right]^{T}$, respectively. Then, the conditional variance of the correlator output is given by the $i$ th effective noise floor

$$
\operatorname{Var}\left[Y^{(i)}[\ell] \mid \boldsymbol{\tau}, \boldsymbol{\nu}\right]=N_{0}+I_{0}^{(i)}(\boldsymbol{\tau}, \boldsymbol{\nu}), \quad i=1, \ldots, P Q
$$

The contribution of MAI is given by

$$
\begin{aligned}
I_{0}^{(i)}(\boldsymbol{\tau}, \boldsymbol{\nu}) & =\sum_{k=2}^{K} I_{k}^{(i)}\left(\tau_{k}, \nu_{k}\right) \\
I_{k}^{(i)}\left(\tau_{k}, \nu_{k}\right) & =P_{k} \operatorname{SSCD}\left(\tau^{(i)}-\tau_{k}, \nu^{(i)}-\nu_{k}\right) .
\end{aligned}
$$

For any $\tau, \nu \in \mathbb{R}$, the SSC-D factorizes as

$$
\operatorname{SSCD}(\tau, \nu)=T_{c} \alpha(\tau) \beta(\nu),
$$

with a pulse interference function $\alpha(\tau)$ and a code interference function $\beta(\nu)$. These functions can be expressed as the Fourier series

$$
\begin{aligned}
& \alpha(\tau)=\alpha_{0}+2 \sum_{m=1}^{\infty} \alpha_{m} \cos \left(2 \pi m \tau / T_{c}\right) \\
& \beta(\nu)=\beta_{0}+2 \sum_{n=1}^{N-1} \beta_{n} \cos \left(2 \pi \nu n T_{0}\right) .
\end{aligned}
$$

The series coefficients are given by

$$
\begin{aligned}
\alpha_{m} & =\frac{1}{T_{c}^{3}} \int_{-\infty}^{\infty}|H(f)|^{2}\left|H\left(\frac{m}{T_{c}}-f\right)\right|^{2} \mathrm{~d} f \\
& =\frac{1}{T_{c}^{3}} \int_{-\infty}^{\infty} \rho_{h}^{2}(t) e^{-\mathrm{j} 2 \pi \frac{m}{T_{c}} t} \mathrm{~d} t \\
\beta_{n} & =\left(1-\frac{n}{M}\right)\left(1-\frac{n}{N}\right), \quad n \leq N \leq M .
\end{aligned}
$$

Proof. A proof for the results (20)-(27) is given in the Appendix.

The SSC-D has several interesting properties, which show that it is a generalization of the SSC:

- Like the standard SSC, the SSC-D has units of seconds (or $1 / \mathrm{Hz}$ ). Rather than a coefficient, it is a function of relative code-phase and relative Doppler frequency.

- The two interference functions are dimensionless and periodic with $T_{c}$ or $T_{0}^{-1}$, respectively. They are shown in Figs. 4, 5.

- The pulse interference function $\alpha(\tau)$ is determined solely by the pulse shape and the fractional part of $\tau / T_{c}$. Computation of the coefficients $\alpha_{m}$ via (26a) is more convenient for band-limited pulse shapes, while (26b) is easier to compute for time-limited pulse shapes. For the common pulse shapes shown in Fig. 3, the first few Fourier coefficients are given in Table I.

- The code interference function $\beta(\nu)$ depends on the fractional part of $\nu T_{0}$, as well as on the number of code repetitions per correlation $(N)$ and per symbol $(M)$. Compared with $\alpha(\tau)$, it can cause much greater variations of the SSC-D. It assumes a maximum of $N+\left(1-N^{2}\right) /(3 M)$ if its argument is a multiple of the code rate. The expression (27) appears to be new to the literature in this form, but the special cases $M=N$ and $M \rightarrow \infty$ are known (e.g. [24]). In all other cases, where $N<M<\infty$, the function graph of $\beta(\nu)$ is between solid and dash-dotted lines of the same color in Fig. 5.

- The standard SSC is simply determined by the mean components of the interference functions as $\mathrm{SSC}=T_{c} \alpha_{0} \beta_{0}$.

- The mean component of the code interference function is always $\beta_{0}=1$, which is why the effect of the important system parameters $M$ and $N$ cannot be reflected by the standard SSC.

- The standard SSC can be interpreted as the expectation of the SSC-D with respect to the uniformly distributed $\tau_{k}$ and $\nu_{k}$, since $\mathrm{E}\left[\alpha\left(\tau^{(i)}-\tau_{k}\right)\right]=\alpha_{0}$ and $\mathrm{E}\left[\beta\left(\nu^{(i)}-\nu_{k}\right)\right]=$ $\beta_{0}$ (assuming that $F_{0} T_{0} \gg 1$ ). Thus, $\operatorname{E}\left[\operatorname{SSCD}\left(\tau^{(i)}-\right.\right.$ $\left.\left.\tau_{k}, \nu^{(i)}-\nu_{k}\right)\right]=\mathrm{SSC}$ and $\mathrm{E}\left[I_{0}^{(i)}(\boldsymbol{\tau}, \boldsymbol{\nu})\right]=I_{0}$.

- There are even some special signals for which the SSC-D is fully equivalent to the SSC. If the pulse shape satisfies $\alpha(\tau) \equiv \alpha_{0}$ (e.g. a RRC pulse with zero roll-off) and the symbol rate equals the code rate $(M=1)$, we have $\operatorname{SSCD}(\tau, \nu) \equiv T_{c} \alpha_{0} \beta_{0}=\mathrm{SSC}=$ const.

We use the name effective noise floor for (20), as this name has been established for interference measures with dimension $\mathrm{W} / \mathrm{Hz}$ that allow the use of standard AWGN performance 


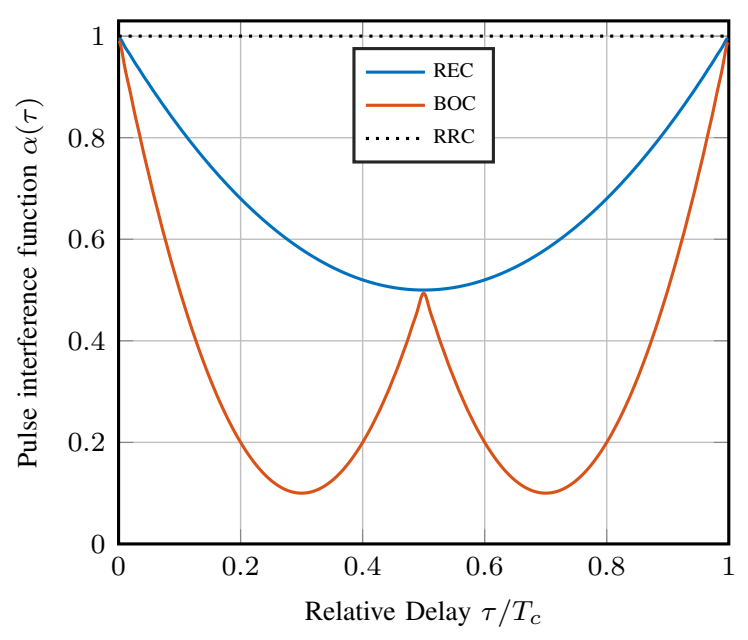

Fig. 4. Pulse interference function vs. relative delay for various pulse shapes: rectangular (REC), binary offset carrier (BOC), root-raised cosine (RRC).

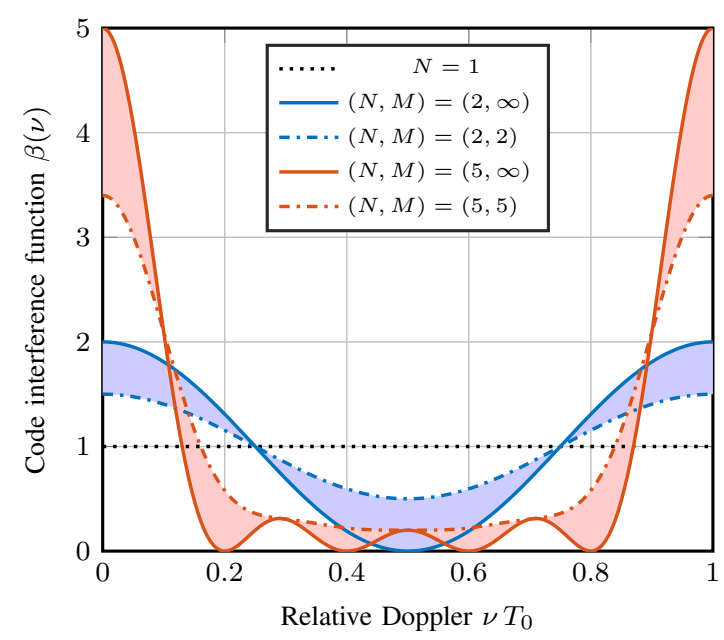

Fig. 5. Code interference function vs. relative Doppler. $N$ and $M$ are the number of code repetitions per coherent correlation and per symbol, respectively. Shaded areas indicate the cases where $N<M<\infty$.

formulas [30], [31]. In particular, we could use the effective noise floors $i=1, \ldots, P Q$ to compute very accurate BPF and GPF for a given $\tau, \nu$ with the scaled $\chi^{2}-\mathrm{CDF}$

$$
\begin{aligned}
& P_{f}^{(i)}(\lambda \mid \boldsymbol{\tau}, \boldsymbol{\nu})=1-F_{Z}\left(\lambda ; N_{0}+I_{0}^{(i)}(\boldsymbol{\tau}, \boldsymbol{\nu})\right) \\
& P_{F}(\lambda \mid \boldsymbol{\tau}, \boldsymbol{\nu})=1-\prod_{i=1}^{P Q} F_{Z}\left(\lambda ; N_{0}+I_{0}^{(i)}(\boldsymbol{\tau}, \boldsymbol{\nu})\right) .
\end{aligned}
$$

The difficulty of that straightforward approach is the necessity to calculate not one, but $P Q$ effective noise floors for any given $\boldsymbol{\tau}, \boldsymbol{\nu}$. Even if the simplifying assumption $\alpha(\tau) \approx \alpha_{0}$ can be made, this will leave the problem of computing $Q$ effective noise floors. The following scenario shows that the effective noise floor can vary considerably from bin to bin.

Example. Consider Fig. 6. A static receiver observes a Walker(24/3/1) constellation [32]. Each in-view satellite transmits a pure pilot signal with PRN code length $N_{c}=341$, RRC

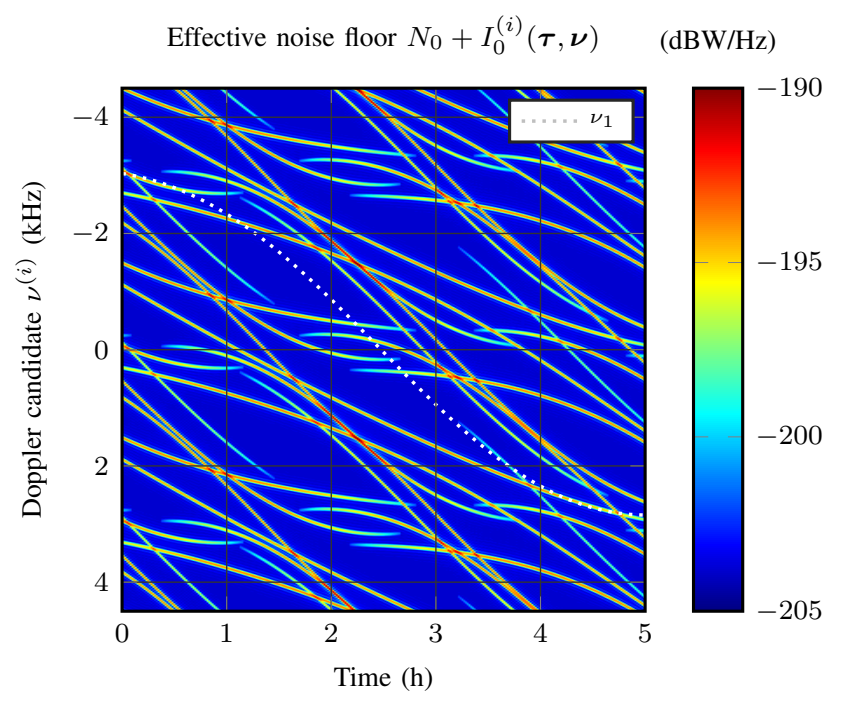

Fig. 6. Variation of the effective noise floor vs. Doppler bins during a satellite pass. The correct Doppler $\nu_{1}$ of the SOI is indicated by the dotted line.

pulse with zero roll-off, and chip rate $1 / T_{c}=1.023 \mathrm{MHz}$. The noise floor $N_{0}$ is $-204 \mathrm{dBW} / \mathrm{Hz}$, and the received powers $P_{k}$ are between $-160 \mathrm{dBW}$ and $-152 \mathrm{dBW}$, using an elevation dependent model [33]. The SOI is transmitted from satellite $k=1$, whose pass takes about five hours and whose Doppler frequency $\nu_{1}$ is indicated by gray dots. The coherent integration time is $20 \mathrm{~ms}$, thus we have $N=60$ code periods per coherent correlation and $M \rightarrow \infty$ (pure pilot). In Fig. 6, the effective noise floor is shown as a function of a bin's Doppler candidate $\nu^{(i)}$ and time. The maximum contribution of interferer $k$ to the effective noise floor is equal to $N P_{k} T_{c} \approx-194 \mathrm{dBW} / \mathrm{Hz}$, and occurs whenever the relative Doppler $\nu^{(i)}-\nu_{k}$ is close to a multiple of $1 / T_{0}=3 \mathrm{kHz}$. This can happen for up to three interferers at the same time for the same Doppler bin, while most other Doppler bins experience an effective noise floor close to the AWGN floor $N_{0}$.

Such a constellation simulation approach is usually computationally unacceptable. It requires $P K$ evaluations of (24) and $Q K$ evaluations of (25), in addition to the costly computation of the overall product (29). Moreover, the performance expressions (28) and (29) are still conditional probabilities. As such, they may be very accurate for an instantaneous satellite constellation, but not representative of all possible $\tau, \nu$.

As a final remark on the SSC-D, we learned that Proposition 1 does not capture some of the more recent findings of Hegarty [24]: he conditioned the effective noise floor on data bit misalignments between interferer and SOI, but averaged over the fractional delay. By contrast, we averaged over the former effect (implicitly, by assuming the stationarized symbol autocorrelation in Fig. 1) but conditioned on the latter. Neither work conditioned on both effects, which could be worth an effort as both appear to be on the order of $3 \mathrm{~dB}$. Nevertheless, the Doppler effect remains the most pronounced by far $(10 \mathrm{~dB}$ and more) and tends to mask the other effects. 


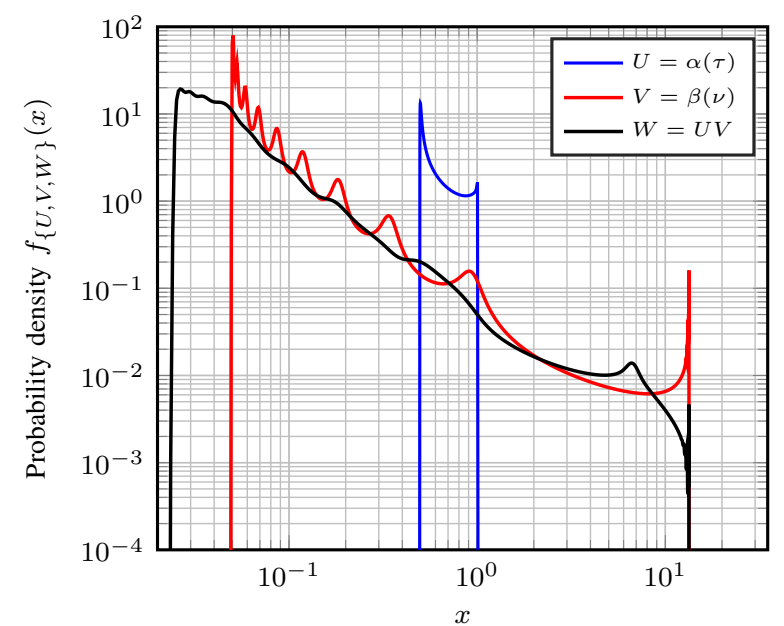

Fig. 7. PDF of pulse interference function, code interference function, and the product of the two, for independent uniformly distributed $\tau, \nu$. (Scenario: REC pulse, $M=N=20$.)

\section{Randomized SSC (SSC-R)}

The SSC-R is an attempt to model the non-uniformity of the effective noise floors, without having to compute each effective noise floor explicitly with the exact conditional formula (20), and replacing them by randomized effective noise floors instead.

We can remove the conditioning on $\tau, \nu$ in the exact conditional performance expressions (28) and (29) to get rid of the dependency on relative code-phases and Doppler frequencies. Consider any bin $i \in\{1, \ldots, P Q\}$. For the BPF, the law of total probability [34] states that the conditioning can be removed by computing

$$
\begin{aligned}
P_{f}^{(i)}(\lambda)= & \mathrm{E}\left[\left(1-F_{Z}\left(\lambda ; N_{0}+I_{0}^{(i)}\right)\right)\right] \\
= & \frac{1}{\left(F_{0} T_{0}\right)^{K-1}} \int_{-F_{0} / 2}^{F_{0} / 2} \ldots \int_{-T_{0} / 2}^{T_{0} / 2} \\
& \quad \times\left(1-F_{Z}\left(\lambda ; N_{0}+I_{0}^{(i)}(\boldsymbol{\tau}, \boldsymbol{\nu})\right)\right) \mathrm{d} \boldsymbol{\tau} \mathrm{d} \boldsymbol{\nu} \\
= & \int_{0}^{\infty}\left(1-F_{Z}\left(\lambda ; N_{0}+x\right)\right) f_{\mathcal{I}_{0}}(x) \mathrm{d} x
\end{aligned}
$$

denoting the PDF of the RV $\mathcal{I}_{0} \triangleq I_{0}^{(i)}(\boldsymbol{\tau}, \boldsymbol{\nu})$ by $f_{\mathcal{I}_{0}}(x)$. Thus we can either compute (30) by a $(2 K-2)$-dimensional integral over all possible $\tau, \boldsymbol{\nu}$ or by a single integral along a PDF. To be able to use the second (and preferable) option, we first need to find the PDF $f_{\mathcal{I}_{0}}(\cdot)$, or an approximation for it. This leads to the notion of the SSC-R.

We propose to approximate $\mathcal{I}_{0}$ by a weighted sum of randomized SSCs

$$
\begin{aligned}
\mathcal{I}_{0} & \approx \sum_{k=2}^{K} P_{k} \mathrm{SSCR}_{k} \\
\mathrm{SSCR}_{k} & \triangleq T_{c} U_{k} V_{k},
\end{aligned}
$$

where $\mathrm{SSCR}_{k}$ and $U_{k}, V_{k}$ are appropriately defined $\mathrm{RVs}$ to emulate the structure of the SSC-D (23). $U_{k}$ and $V_{k}$ are mutually independent and, for $k=2 \ldots, K$, i.i.d. according

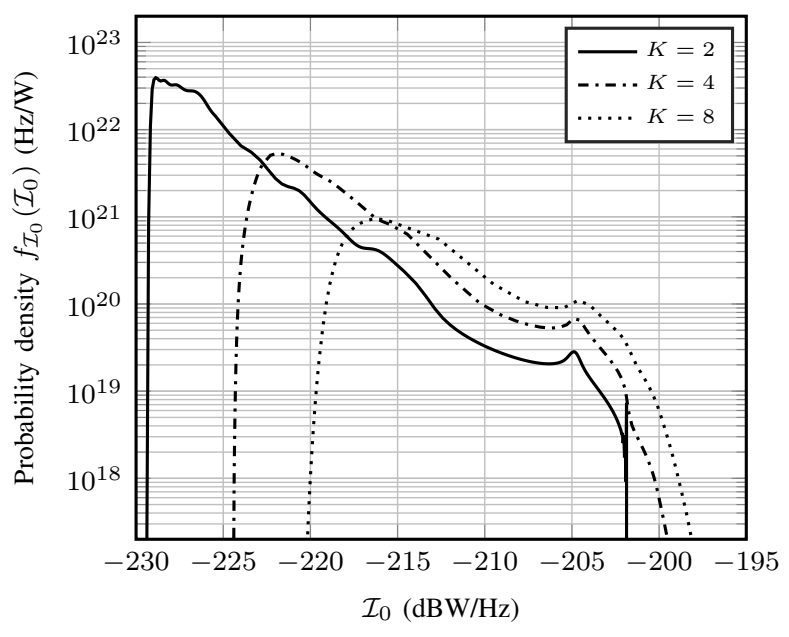

Fig. 8. PDF of the MAI variance $\mathcal{I}_{0}$ for $K-1$ interferers. (Scenario: REC pulse, $1 / T_{c}=1.023 \mathrm{MHz}, P_{2}=\ldots=P_{K}=-153 \mathrm{dBW}, M=N=20$.)

to PDFs $f_{U}(\cdot), f_{V}(\cdot)$, respectively. Then the PDF $f_{\mathcal{I}_{0}}(\cdot)$ can be constructed as follows:

- Starting from the RVs $U \triangleq \alpha(\tau)$ and $V \triangleq \beta(\nu)$ for uniformly distributed $\tau \in\left[0, T_{c}\right)$ and $\nu \in\left[0, T_{0}^{-1}\right)$, compute the PDFs $f_{U}(x), f_{V}(x)$ for all possible realizations $x \geq 0$, using the law of transformation of RVs [34]. The result is shown in Fig. 7.

- Compute the PDF $f_{W}(\cdot)$ of the product $W \triangleq U V$. Using that $U$ and $V$ are independent [34],

$$
f_{W}(x)=\int_{0}^{\infty} f_{V}(\xi) f_{U}(x / \xi) \frac{1}{\xi} \mathrm{d} \xi, \quad x \leq 0 .
$$

The result is shown in Fig. 7.

- The PDF of the SSC-R is the scaled version of $f_{W}(x)$

$$
f_{\mathrm{SSCR}}(x)=\frac{f_{W}\left(\frac{x}{T_{c}}\right)}{T_{c}} .
$$

- The PDF of the weighted sum (31) of independent RVs is obtained by the convolutions

$$
f_{\mathcal{I}_{0}}(x)=\frac{f_{\mathrm{SSCR}}\left(\frac{x}{P_{2}}\right)}{P_{2}} * \ldots * \frac{f_{\mathrm{SSCR}}\left(\frac{x}{P_{K}}\right)}{P_{K}}
$$

An exemplary result for $K=2,4,8$ satellites is shown in Fig. 8.

Note that the PDF $f_{\mathcal{I}_{0}}(x)$ represents the distribution of aggregate MAI as experienced by a specific user antenna and frontend. In particular, any elevation-dependent user antenna gain pattern must be reflected in the weights $P_{k}$. There are of course other ways than (31)-(35) to construct $f_{\mathcal{I}_{0}}(x)$. While the above approach is matched to the system model from Section II, it is also possible to compute an empirical PDF $f_{\mathcal{I}_{0}}(x)$ via measurements or constellation simulation. This would even allow for more sophisticated scenarios, including nonindependent Doppler frequencies, fading, or a varying number of in-view satellites. For instance, we could choose to calculate the SSC-D explicitly during a GNSS constellation period of interest, compute weights $P_{k}$ according to satellite elevation and antenna gain patterns, and then compute a single histogram 
of $I_{0}^{(i)}(\boldsymbol{\tau}, \boldsymbol{\nu})$ over the entire considered constellation period and all search bins $i=1, \ldots, P Q$. Depending on whether the observed constellation period is short-term or long-term, the PDF will be more representative of an instantaneous scenario or of many possible scenarios.

The obtained density $f_{\mathcal{I}_{0}}(x)$ can now be used to compute the BPF via the simple integral in (30). Note that this expression leads to the same BPF regardless of the bin index $i=1, \ldots, P Q$. We have effectively modeled the distribution of any statistic $Z^{(i)}$ by compounding the distributions (10) and (35). If we approximate the statistics as not only identically distributed but also independent across bins, we finally obtain the desired SSC-R based approximation for the GPF

$$
P_{F}(\lambda)=1-\left(\int_{0}^{\infty} F_{Z}\left(\lambda ; N_{0}+\mathcal{I}_{0}\right) f_{\mathcal{I}_{0}}\left(\mathcal{I}_{0}\right) \mathrm{d} \mathcal{I}_{0}\right)^{P Q}
$$

The similarity to the SSC-based GPF becomes obvious when we rewrite the standard result (19), using $I_{0}=\mathrm{E}\left[\mathcal{I}_{0}\right]$, as

$$
P_{F}(\lambda)=1-\left(F_{Z}\left(\lambda ; N_{0}+\int_{0}^{\infty} \mathcal{I}_{0} f_{\mathcal{I}_{0}}\left(\mathcal{I}_{0}\right) \mathrm{d} \mathcal{I}_{0}\right)\right)^{P Q}
$$

While both expressions are approximations of the true GPF, the SSC-R is expected to be much better suited for most relevant scenarios, as it does not assume $P Q$ identical effective noise floors $N_{0}+I_{0}$ across all bins, but only $P Q$ i.i.d. realizations of a random effective noise floor $N_{0}+\mathcal{I}_{0}$.

\section{Probability of Detection}

Let $H_{1}$ be the true hypothesis, i.e., the SOI is present. We say that global detection occurs if the receiver decides for $H_{1}$ and returns the correct bin $i=1$. The probability of this event is called global probability of detection (GPD). It is denoted by $P_{D}^{\downarrow}(\lambda)$ for serial search and by $P_{D}^{\|}(\lambda)$ for parallel search, respectively.

\section{A. Conditional GPD}

We define the $i$ th noncentrality energy as the energy that is delivered to the statistic $Z^{(i)}$ in the absence of MAI and noise

$$
\left.\mathcal{E}^{(i)} \triangleq Z^{(i)}\right|_{\substack{N_{0}=0 \\ K=1}}, \quad i=1, \ldots, P Q
$$

Ideally, we would expect $\mathcal{E}^{(1)}=P_{1} L T$ and $\mathcal{E}^{(2)}=\ldots=$ $\mathcal{E}^{(P Q)}=0$. In practice, this is not the case: energy is lost in the correct bin and can leak into other bins due to various effects. In fact, the noncentrality energies are functions of $\tau_{1}, \nu_{1}$ and the states of the symbol sequence $\boldsymbol{b}_{1} \triangleq\left[b_{1}[0], \ldots, b_{1}[L N]\right]^{T}$ during the observation time. Therefore, $\mathcal{E}^{(1)}, \ldots, \mathcal{E}^{(P Q)}$ are statistically dependent RVs. Exemplarily, we determined the marginal distribution of the noncentrality energy $\mathcal{E}^{(1)}$ numerically in Fig. 9. It can be shown that [7]

$$
\mathcal{E}^{(i)}=\alpha_{C}\left(\tau^{(i)}-\tau_{1}\right) \beta_{C}\left(\nu^{(i)}-\nu_{1} ; \vartheta_{1} T_{0}+\tau_{1}, \boldsymbol{b}_{1}\right) P_{1} L T,
$$

with a code-phase correlation function

$$
\alpha_{C}(\tau)=\sum_{i=-\infty}^{\infty} \frac{\rho_{h}^{2}\left(\tau-i T_{0}\right)}{T_{c}^{2}}
$$

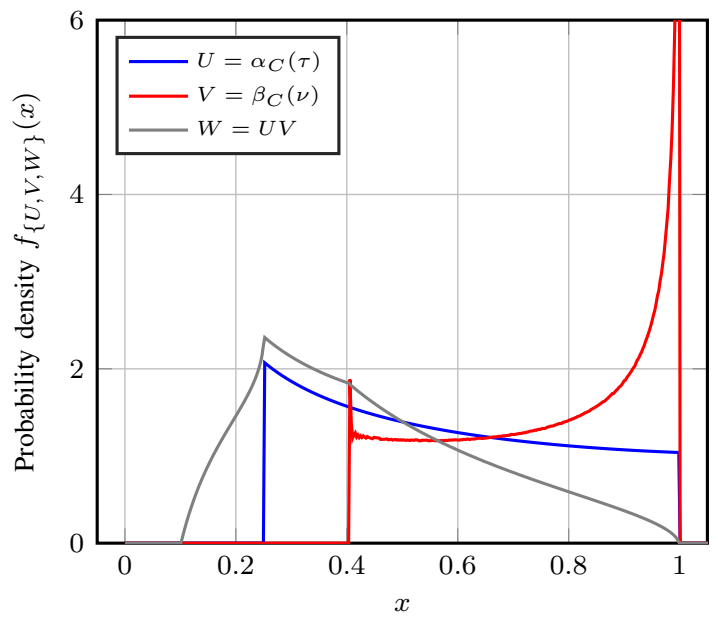

Fig. 9. PDF of the energy loss in the correct bin with code-phase spacing $\Delta \tau=T_{c}$ and Doppler spacing $\Delta \nu=1 / T$, for a REC pulse and pure pilot signaling $(\boldsymbol{b}=\mathbf{1})$.

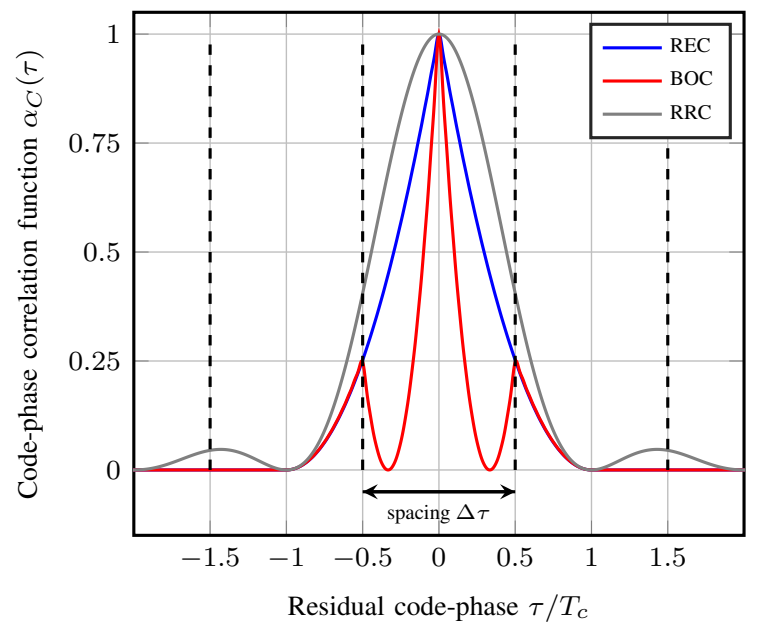

Fig. 10. Correlation vs. code-phase for various pulse shapes: rectangular (REC), binary offset carrier (BOC), root-raised cosine (RRC) with zero rolloff.

and a Doppler correlation function

$$
\beta_{C}(\nu ; \theta, \boldsymbol{b})=\frac{T_{c}^{2}}{T^{2}} \frac{\sin ^{2}(\pi \nu T)}{\sin ^{2}\left(\pi \nu T_{c}\right)}\left(1-\frac{2 X(\boldsymbol{b})}{L} \phi(\nu, \theta)\right),
$$

with the number of symbol transitions $X(\boldsymbol{b}) \in\{0, \ldots, L\}$ and an auxiliary function ${ }^{3}$ [7]

$$
\phi(\nu, \theta)=\cot ^{2}(\pi \nu T)\left(\tan (\pi \nu T) \sin (2 \pi \nu \theta)-2 \sin ^{2}(\pi \nu \theta)\right) .
$$

If a pure pilot signal is considered, we can simply use $\beta_{C}(\nu) \triangleq$ $\left(T_{c} / T\right)^{2}\left(\sin (\pi \nu T) / \sin \left(\pi \nu T_{c}\right)\right)^{2}$. The energy loss and leakage effects are illustrated in Figs. 10, 11.

Unlike MAI and noise, the contribution of the SOI to the statistic $Z^{(i)}$ is not Gaussian distributed. As proposed by [7], [35], we model this contribution by modifying the central $\chi^{2}$ distribution from (10) to a noncentral $\chi^{2}$-distribution with

\footnotetext{
${ }^{3}$ Note that the auxiliary function becomes more complicated if $L>1$ and $N<M$, a case which is ommitted at this point for brevity but is treated in the thorough work of O'Driscoll [7].
} 


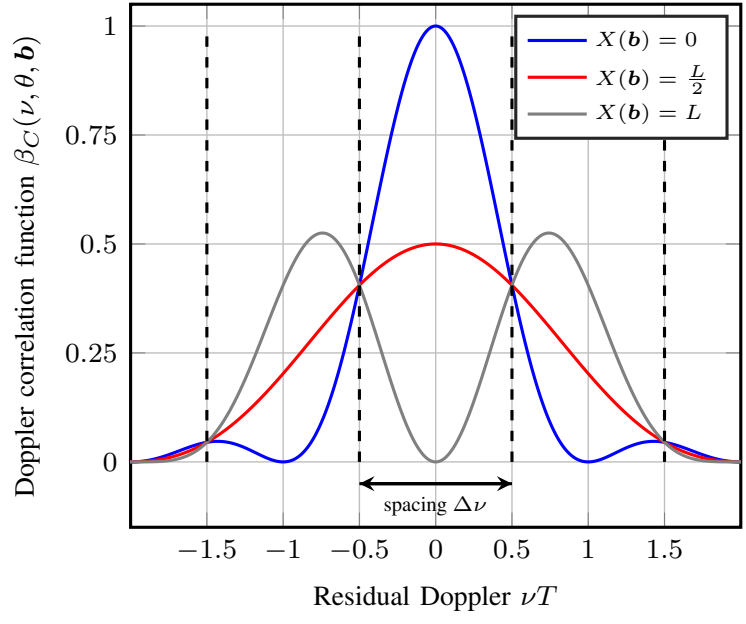

Fig. 11. Correlation vs. Doppler, with symbol boundaries in the middle of $L$ coherent subintervals $(\theta=T / 2)$. The number of symbol transitions is $X(\boldsymbol{b}) \in\{0,1, \ldots, L\}$.

random noncentrality parameter (hence, a compound probability distribution). MAI and noise are the same as under $H_{0}$ and can be modeled by either of the previously discussed models (Sections III-A-III-D), which we represent by a generic effective noise floor $\mathcal{N}_{0}^{(i)}$ at this point. Adding the noncentral component to (10) leads to the generic CDF of $Z^{(i)}$ under $H_{1}$, given $\mathcal{E}^{(i)}$ and $\mathcal{N}_{0}^{(i)}$,

$F_{Z}^{\prime}\left(z ; \mathcal{E}^{(i)}, \mathcal{N}_{0}^{(i)}\right) \triangleq 1-Q_{L}\left(\sqrt{2 \frac{\mathcal{E}^{(i)}}{\mathcal{N}_{0}^{(i)}}}, \sqrt{2 \frac{z}{\mathcal{N}_{0}^{(i)}}}\right), z \geqslant 0$.

Here, $Q_{L}(\cdot, \cdot)$ denotes the $L$ th order Marcum Q-function [36]. This CDF is a scaled version of the CDF of a noncentral $\chi^{2}$-distribution with $2 L$ degrees of freedom and noncentrality parameter $2 \mathcal{E}^{(i)} / \mathcal{N}_{0}^{(i)}$. The according (yet conditional) bin probability of detection (BPD) is

$$
P_{d}^{(i)}(\lambda)=1-F_{Z}^{\prime}\left(\lambda ; \mathcal{E}^{(i)}, \mathcal{N}_{0}^{(i)}\right) .
$$

This probability is a compound probability in $\mathcal{E}^{(i)}$ and, depending on which SSC model is used, in $\mathcal{N}_{0}^{(i)}$.

Next, we compute the conditional GPD, given the vector of all noncentrality energies $\mathcal{E} \triangleq\left[\mathcal{E}^{(1)}, \ldots, \mathcal{E}^{(P Q)}\right]^{T}$ and the vector of effective noise floors $\mathcal{N}_{0} \triangleq\left[\mathcal{N}_{0}^{(1)}, \ldots, \mathcal{N}_{0}^{(P Q)}\right]^{T}$. Well-known formulas [26] lead to the following results for serial or parallel search

$$
P_{D}^{\downarrow} \mid \mathcal{E}, \mathcal{N}_{0}=\frac{1-F_{Z}^{\prime}\left(\lambda ; \mathcal{E}^{(1)}, \mathcal{N}_{0}^{(1)}\right)}{P Q} \sum_{j=1}^{P Q} \prod_{i=2}^{j} F_{Z}^{\prime}\left(\lambda ; \mathcal{E}^{(i)}, \mathcal{N}_{0}^{(i)}\right)
$$

$$
P_{D}^{\|} \mid \mathcal{E}, \mathcal{N}_{0}=\int_{\lambda}^{\infty} f_{Z}^{\prime}\left(z ; \mathcal{E}^{(1)}, \mathcal{N}_{0}^{(1)}\right) \prod_{i=2}^{P Q} F_{Z}^{\prime}\left(z ; \mathcal{E}^{(i)}, \mathcal{N}_{0}^{(i)}\right) \mathrm{d} z
$$

as long as we condition on $\mathcal{E}$ and $\mathcal{N}_{0}$. It remains to remove the conditioning on these parameters.

To simplify computation of the above equations, we assume $\mathcal{E}^{(i)} \approx 0$ if $\left|\tau^{(i)}-\tau_{1}\right|>T_{c}$ and $\left|\nu^{(i)}-\nu_{1}\right|>3 /(2 T)$.
Therefore, apart from the the correct bin and some few adjacent bins, most bins are treated as central $\chi^{2}$-distributed.

\section{B. Removing the conditioning}

Starting from the conditional GPD (45) or (46), respectively, we remove the conditioning as follows.

1) Apply an SSC model:

- To use the standard SSC, simply set $\mathcal{N}_{0}^{(i)}=N_{0}+I_{0}$ for $i=1, \ldots, P Q$.

- To use the SSC-D, simply set $\mathcal{N}_{0}^{(i)}=N_{0}+I_{0}^{(i)}(\boldsymbol{\tau}, \boldsymbol{\nu})$ for $i=1, \ldots, P Q$.

- To use the SSC-R, apply the law of total probability w.r.t. $\mathcal{N}_{0}$ : replace the conditional $\mathrm{CDF}$ $F_{Z}^{\prime}\left(z ; \mathcal{E}^{(i)}, \mathcal{N}_{0}^{(i)}\right)$ by the partly conditional $\mathrm{CDF}$

$$
F_{Z}^{\prime}\left(z ; \mathcal{E}^{(i)}\right) \triangleq \int_{0}^{\infty} F_{Z}^{\prime}\left(z ; \mathcal{E}^{(i)}, N_{0}+x\right) f_{\mathcal{I}_{0}}(x) \mathrm{d} x
$$

for all bins. For parallel search and $i=1$, replace the conditional PDF with $f_{Z}^{\prime}\left(z ; \mathcal{E}^{(1)}\right) \triangleq \frac{\mathrm{d}}{\mathrm{d} z} F_{Z}^{\prime}\left(z ; \mathcal{E}^{(1)}\right)$.

2) Remove the conditioning on $\mathcal{E}$ directly by averaging over the random SOI parameters $\tau_{1}, \nu_{1}$ and (if applicable) the possible symbol sequences $\boldsymbol{b}_{1},{ }^{4}$ using (39) as in [7].

3) In case the SSC-D was used, average over the remaining random parameters $\tau, \nu$.

\section{NUMERICAL RESUlTS}

Unless stated otherwise, the following baseline setup is used for the remainder of this work.

- REC pulse, chip rate $1 / T_{c}=1.023 \mathrm{MHz}$;

- symbol rate $1 / T_{b}=0$ (pure pilot);

- code length $N_{c}=341 \Rightarrow$ code period $T_{0}=0.33 \mathrm{~ms}$;

- coh. integration time $T=1 \mathrm{~ms} \Rightarrow N=3$ code periods;

- number of noncoherent summations $L=1$;

- Doppler span $F_{0}=8 \mathrm{kHz}$

- number of satellites $K=8$;

- noise floor $N_{0}=-204 \mathrm{dBW} / \mathrm{Hz}$;

- code-phase spacing $\Delta \tau=T_{c}$;

- Doppler spacing $\Delta \nu=1 / T$.

This setup leads to $P Q=341 \times 8=2728$ bins. Furthermore, we consider three characteristic scenarios, which are represented by three different power profiles $\left(P_{1}, \ldots, P_{K}\right)$ :

1) Balanced scenario: maximum received power [25] for all satellites: $P_{k}=-153 \mathrm{dBW}$ for $k=1, \ldots, K$.

2) Near-far scenario: maximum received power for $k=$ $2, \ldots, K$, minimum received power [25] for the SOI: $P_{1}=-158.5 \mathrm{dBW}$.

3) Weak signals scenario: very low received power for all signals: $P_{k}=-180 \mathrm{dBW}$ for $k=1, \ldots, K$.

As per definition, $P_{1}=0$ always under $H_{0}$. Note that the power profiles of Scenarios 1 and 2 are within system specifications [25], while only few systems are committed to

\footnotetext{
${ }^{4}$ There are $(L+1) M$ possible symbol sequences, as $\boldsymbol{b}$ can be parameterized in terms of the number of symbol transitions $X(\boldsymbol{b}) \in\{0, \ldots, L\}$ and the uniformly random initial symbol-phase $\vartheta_{1} \in\{1, \ldots, M\}$. If $L>1, X(\boldsymbol{b})$ follows a binomial distribution with $L$ trials and success probability $1 / 2$.
} 


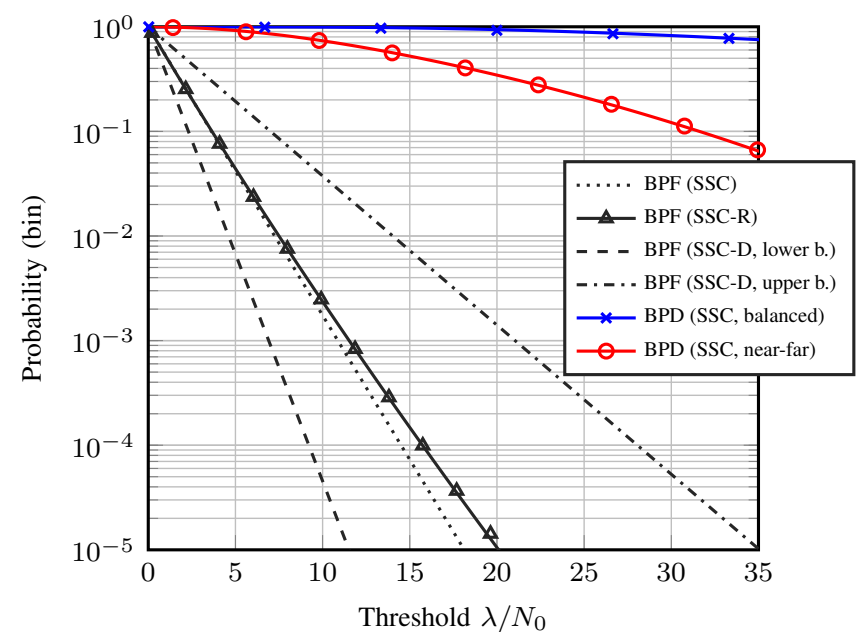

Fig. 12. Bin probabilities vs. threshold for Scenarios 1 (balanced) and 2 (near-far) with baseline setup $\left(N_{c}=341, T=1 \mathrm{~ms}\right)$. Markers represent simulation results.

service in weak signal conditions such as Scenario 3 [37]. However, these conditions are of some interest for navigation in space [12] or indoors [38].

\section{A. Baseline Setup}

Fig. 12 shows the BPF that is obtained with the baseline setup in Scenarios 1 and 2. In fact, there is not one $\mathrm{BPF}$ but many, depending on the bin's effective noise floor. The simulation results (markers) were obtained by Monte-Carlo simulations, randomly selecting one of the $P Q$ bins and determining its BPF. The BPF obtained with the SSC-R is representative not for any particular bin, but for the ensemble of bins, and matches well with the simulation results. The standard SSC leads to a slight underestimation of the BPF. For the SSC-D, only the BPF of the bin with the highest and lowest effective noise floor are shown. It is interesting to note that the BPFs under SSC and SSC-D appear as straight lines in a semilogarithmic plot, as the BPF for $L=1$ is an exponential function (10) of the threshold. By contrast, the SSC-R leads to a compound (mixture) BPF and appears as a slightly curved line. For the BPD in bin $i=1$, all SSC models lead to essentially the same results.

Fig. 13 shows the global probabilities $P_{F}(\lambda), P_{D}^{\|}(\lambda), P_{D}^{\downarrow}(\lambda)$ as a function of the threshold, for Scenarios 1 and 2 . As these scenarios differ only in terms of the SOI power, $P_{F}(\lambda)$ is the same in either case, while detection is less likely in the near-far scenario. It can be observed that the standard SSC underestimates the GPF and slightly overestimates the GPD for all thresholds, while the SSC-R is in line with results from Monte-Carlo simulations. ${ }^{5}$ Plotting the GPD against the GPF, with the threshold $0 \leqslant \lambda<\infty$ as the varying parameter, leads to the ROC curve in Fig. 14. The receiver can operate at any point on the ROC curve by choosing the threshold accordingly.

${ }^{5}$ Note that we were not able to report any GPF values obtained via the SSC-D, since the evaluation of the product (29) and subsequent averaging with respect to $\tau, \nu$ indeed turned out to be too computationally burdensome.

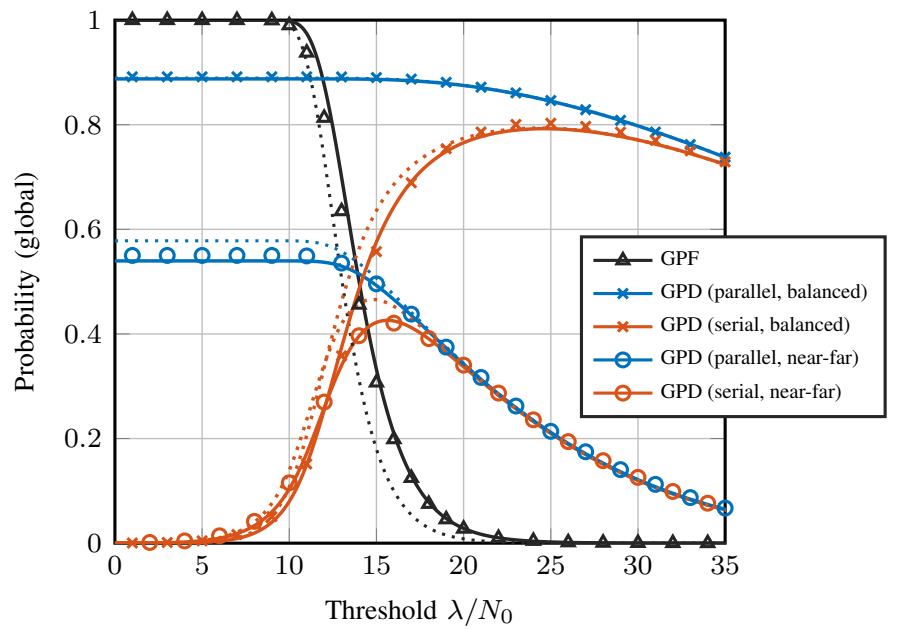

Fig. 13. Global probabilities vs. threshold for Scenarios 1 (balanced) and 2 (near-far) with baseline setup $\left(N_{c}=341, T=1 \mathrm{~ms}\right)$. Solid lines: SSC-R, dotted lines: SSC, markers: simulation results. $P Q=341 \times 8$ bins.

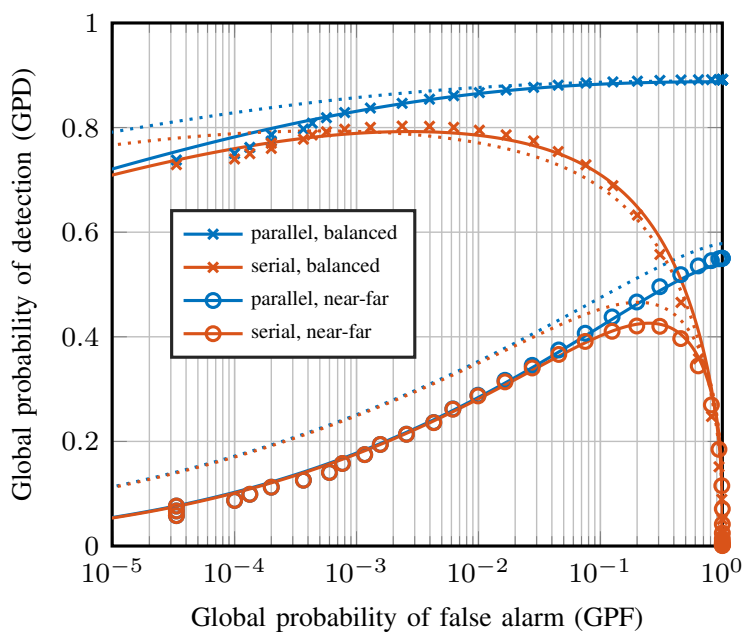

Fig. 14. ROC curve for Scenarios 1 (balanced) and 2 (near-far) with baseline setup $\left(N_{c}=341, T=1 \mathrm{~ms}\right)$. Solid lines: SSC-R, dotted lines: SSC, markers: simulation results. $P Q=341 \times 8$ bins.

\section{B. Increasing the coherent integration time $T$}

Increasing the coherent integration time is a good receiver side solution to enhance the acquisition reliability, especially in a near-far scenario. This leads to a proportional increase of the number of Doppler bins $Q$, while the number of codephase bins $P$ remains constant. As the code length $N_{c}$ and code period $T_{0}$ remain fixed, the receiver performs coherent integration over multiple code periods $N$. Increasing $N$ reveals the great weakness of the standard SSC: it depends only on $T$ but not on $N=T / T_{0}$. Thus, for large values of $N$, the BPF is grossly underestimated by the standard SSC, but correctly modeled by the SSC-R, as is shown in Fig. 15. The standard SSC also leads to a very overoptimistic ROC for $T=5 \mathrm{~ms}$ and $N=15$ in Fig. 16. 


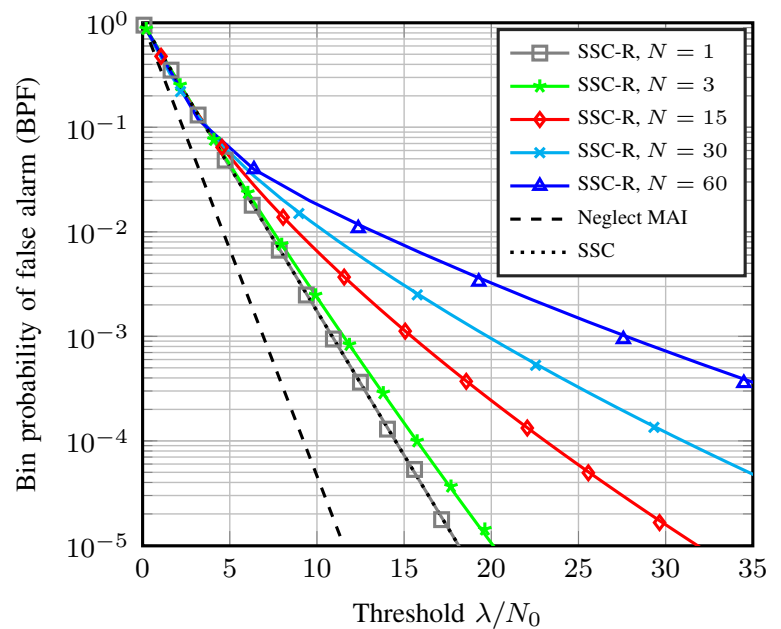

Fig. 15. BPF vs. threshold for Scenarios 1 (balanced) and 2 (near-far) with varying number $N$ of code periods per coherent integration. Markers represent simulation results.

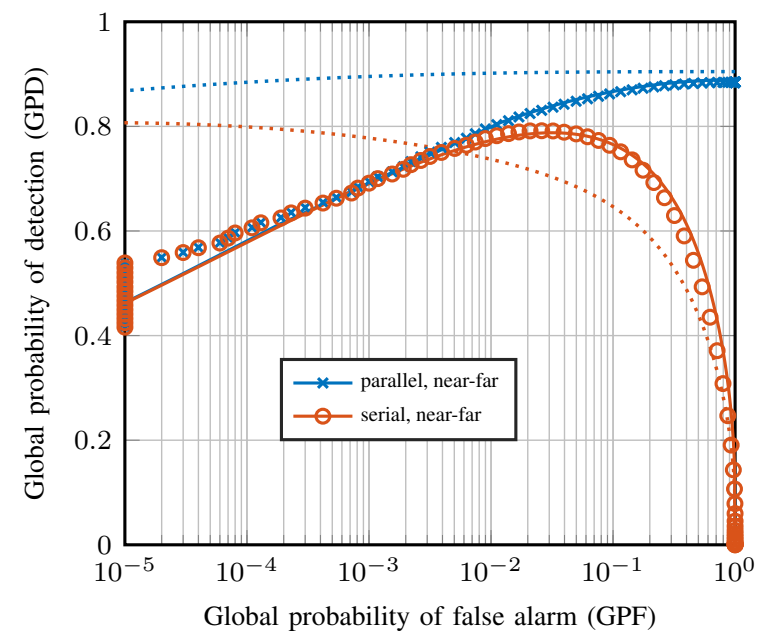

Fig. 16. ROC for Scenario 2 (near-far) with extended coherent integration time, $N_{c}=341, T=5 \mathrm{~ms}, N=15$. Solid lines: SSC-R, dotted lines: SSC, markers: simulation results. $P Q=341 \times 40$ bins.

\section{Increasing the number of noncoherent summations $L$}

For weak signals such as in Scenario 3, a good option to enhance reliability further is to increase the number of noncoherent summations $L$. Increasing the coherent integration time $T$ further and further would lead to an excessive number of Doppler bins, and is also difficult due to limitations of the receiver clock stability of mass-market devices. Using more than $T=20 \mathrm{~ms}$ (hence $N=60$ in this case) is usually not practical. In Fig. 17, we show the ROC for $N=60$ and $L=1,5,10,20,30$. It can be observed that despite the large value of $N$, the standard SSC is already accurate (and virtually coincides with the SSC-R). This is due to the very low relative threshold $\lambda / N_{0}$ at the relevant operating points. As could be observed from Fig. 15, SSC and SSC-R lead to similar results as long as the ratio $\lambda / N_{0}$ is small.

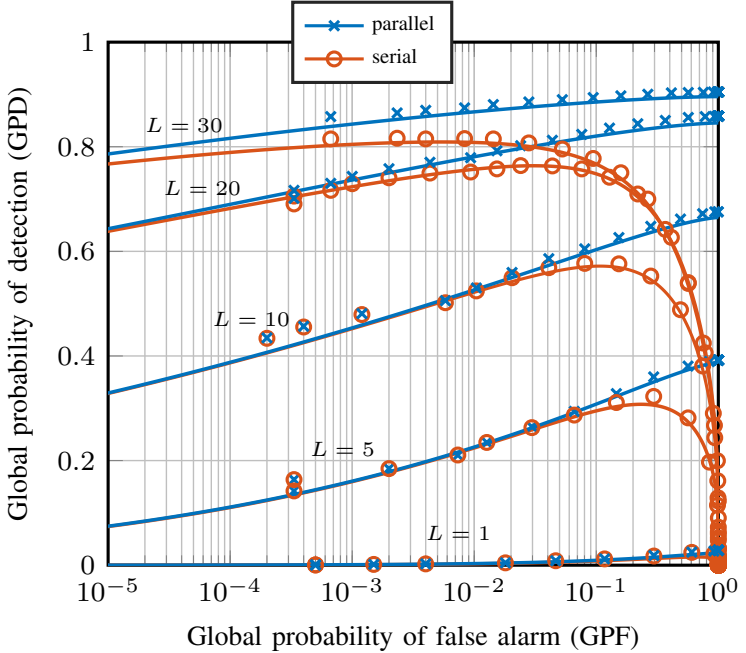

Fig. 17. ROC for Scenario 3 (weak signals) with $N=60$ coherent integrations and $L$ noncoherent summations. Solid lines: SSC, markers: simulation results. $P Q=341 \times 160$ bins.

\section{SignAl DESIGN: MINIMIZING PRN CODE LENGTH}

As an application example of the proposed methodology, we consider the design of an acquisition signal for the European GNSS Galileo. This signal would complement the existing Galileo Open Service signals E1-B and E1-C transmitted at $1575.42 \mathrm{MHz}$ [25] and will be called E1-D in the following. E1-B and E1-C use a PRN code length of $N_{c}=4092$, which is why we consider integer divisors $N_{c}=2046,1023,682,372, \ldots$ as possible E1-D code lengths: this would allow for an easier handover to the signals with longer PRN code. In terms of symbol rate, we consider the two options of a "pure pilot" signal and a "quasi-pilot" [14] signal with symbol duration $M T_{0}=20 \mathrm{~ms}$. The pulse shape is $\mathrm{REC}$ with chipping rate $1 / T_{c}=1.023 \mathrm{MHz}$.

We aim to minimize the PRN code length over the set of integer divisors of 4092, while ensuring that the target reliability of $P_{D}^{\|}(\lambda)>80 \%, P_{F}(\lambda)<5 \%$ can be achieved for some $\lambda \geqslant 0$. The coherent integration time is $T=4 \mathrm{~ms}$, while $L=1$. Interferers $k=2, \ldots, 8$ are received with maximum power $P_{k}=-153 \mathrm{dBW}$. In Fig.18 (presented at [39]), we show the sensitivity, i.e., the necessary received power $P_{1}$ at which reliable acquisition is possible. For a PRN code length to be feasible, the sensitivity should not be above the nominal minimum received power level of $-158.5 \mathrm{dBW}$ [25]. As a result, we suggest the minimum code length of $N_{c}=341$ for a pure pilot signal or, alternatively, $N_{c}=682$ for a quasi-pilot signal with $50 \mathrm{~Hz}$ symbol rate. The slightly worse sensitivity of the quasi-pilot compared with a pure pilot is due to symbol transititons that occasionally lead to an energy loss. Note that the standard SSC is accurate only if $T=T_{0}$ and is therefore of no use for this application.

\section{CONCLUSION}

We argued in the introduction that signals with short PRN code and low symbol rate are an attractive option to facilitate rapid, low energy acquisition, but are vulnerable to MAI. 


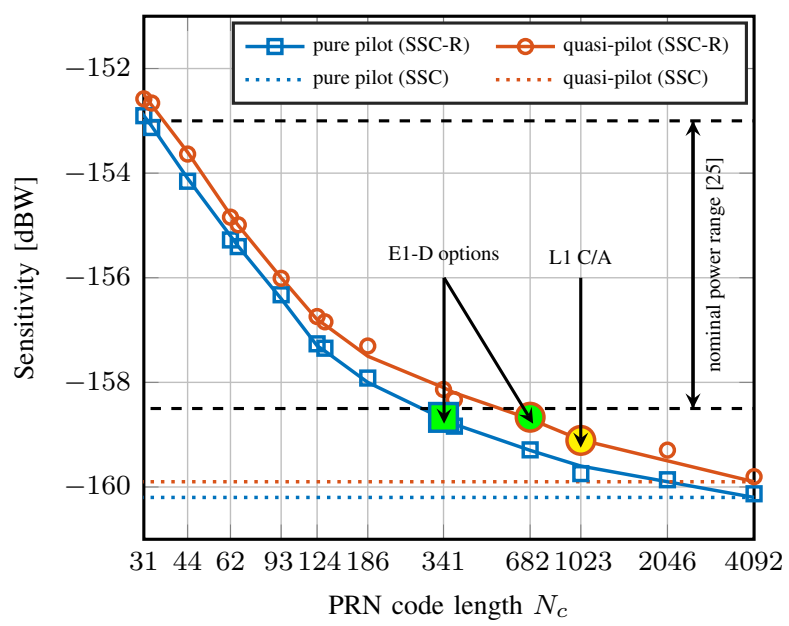

Fig. 18. Sensitivity of possible Galileo E1-D signals vs. PRN code length, for a pure pilot $(M \rightarrow \infty)$ and a quasi-pilot $(M=20)$ signal. The proposed E1-D options and GPS L1 C/A are indicated. Solid lines: SSC-R, dotted lines: SSC, markers: simulation results.

We demonstrated that some few Doppler search bins can be severely affected by MAI, and are thus more likely to lead to a global false alarm in the acquisition search. We proposed a methodology that can be used to assess the acquisition reliability in terms of the ROC curve (GPF plotted vs. GPD). The methodology is general and simple enough to be used in a signal design approach, where PRN code length, symbol rate, and other signal characteristics are flexible parameters to be selected. Applying the methodology to a signal design example, we showed that PRN code lengths on the order of 300-700 are feasible options for a dedicated acquisition signal for Galileo, which would reduce the number of search bins by a factor of $1.5-3$ as compared with GPS L1 C/A. As an outlook, radio frequency compatibility of such a signal with co-existing signals in the same frequency band should be assessed, using models to assess cyclostationary intersystem MAI [22].

\section{ACKNOWLEDGMENT}

We would like to thank the anonymous reviewers whose comments helped improve this manuscript.

\section{APPENDIX}

\section{DERIVATION OF SSC AND SSC-D}

For the following calculations, it is useful to define the $\ell$ th segment of the local replica

$$
x_{\ell}^{(i)}(t) \triangleq e^{\mathrm{j} 2 \pi \nu^{(i)}} t \sum_{n=\ell N}^{\ell N+N-1} s_{1}\left(t-n T_{0}-\tau^{(i)}\right),
$$

for segments $\ell=0, \ldots, L-1$ and bins $i=1, \ldots, P Q$, and then rewrite the complex correlator output as

$$
\begin{aligned}
Y^{(i)}[\ell] & =\frac{1}{\sqrt{T}} \int_{\ell T}^{(\ell+1) T} \overline{x^{(i)}(t)} r(t) \mathrm{d} t \\
& \approx \frac{1}{\sqrt{T}} \int_{-\infty}^{\infty} \overline{x_{\ell}^{(i)}(t)} r(t) \mathrm{d} t .
\end{aligned}
$$

Note that this approximation is exact if the code waveform is strictly time-limited to $\left(-T_{0} / 2, T_{0} / 2\right)$, e.g. if a REC or BOC pulse shape is used. For band-limited waveforms, the approximation is still reasonably accurate and facilitates the analysis considerably.

We begin by expressing the correlator output as the superposition of $K$ signal contributions plus noise

$$
\begin{aligned}
Y^{(i)}[\ell] & =\sum_{k=1}^{K} X_{k}^{(i)}[\ell]+W^{(i)}[\ell] \\
X_{k}^{(i)}[\ell] & =\frac{1}{\sqrt{T}} \int_{-\infty}^{\infty} \overline{x_{\ell}^{(i)}(t)} x_{k}(t) \mathrm{d} t \\
W^{(i)}[\ell] & =\frac{1}{\sqrt{T}} \int_{-\infty}^{\infty} \overline{x_{\ell}^{(i)}(t)} w(t) \mathrm{d} t .
\end{aligned}
$$

The noise contribution has mean zero and satisfies

$$
\begin{aligned}
& \mathrm{E}\left[\overline{W^{(i)}[\ell]} W^{(i)}\left[\ell^{\prime}\right]\right] \\
& =\frac{1}{T} \int_{-\infty}^{\infty} \int_{-\infty}^{\infty} \mathrm{E}[\overline{w(t)} w(u)] \mathrm{E}\left[x_{\ell}^{(i)}(t) \overline{\left.x_{\ell}^{(i)}(u)\right]} \mathrm{d} u \mathrm{~d} t\right. \\
& =\frac{1}{T} \sum_{n=\ell N}^{\ell N+N-1} \sum_{\ell^{\prime}=\ell^{\prime} N}^{N+N-1} \sum_{j=0}^{N_{c}-1} \sum_{j^{\prime}=0}^{N_{c}-1} \mathrm{E}\left[c_{1}[j] c_{1}\left[j^{\prime}\right]\right] \\
& \quad \times \int_{-\infty}^{\infty} \int_{-\infty}^{\infty} \mathrm{d} u \mathrm{~d} t N_{0} \delta(t-u) e^{\mathrm{j} 2 \pi \nu^{(i)}(t-u)} \\
& \quad \times h\left(t-j T_{c}-n T_{0}-\tau^{(i)}\right) h\left(u-j^{\prime} T_{c}-n^{\prime} T_{0}-\tau^{(i)}\right) \\
& =\frac{N_{0}}{T} \delta\left[\ell-\ell^{\prime}\right] \sum_{n=\ell N}^{\ell N+N-1} \sum_{j=0}^{N_{c}-1} \rho_{h}(0) \\
& =\delta\left[\ell-\ell^{\prime}\right] N_{0} .
\end{aligned}
$$

As $w(t)$ is complex AWGN, it follows that $W^{(i)}[0], \ldots, W^{(i)}[L-1]$ are i.i.d. CSCG RVs with mean zero and variance $N_{0}$.

The MAI terms $X_{k}^{(i)}[\ell]$ for $k=2, \ldots, K$ have mean zero, since AWGN and interfering signals are independent and have both mean zero. The conditional variance of the MAI terms can be derived as follows,

$$
\begin{aligned}
& I_{k}^{(i)}\left(\tau_{k}, \nu_{k}\right) \\
& \triangleq \operatorname{Var}\left[X_{k}^{(i)}[\ell] \mid \tau_{k}, \nu_{k}\right] \\
& =\frac{1}{T} \int_{-\infty}^{\infty} \int_{-\infty}^{\infty} \mathrm{E}\left[\overline{x_{k}(t)} x_{k}(u) \mid \tau_{k}, \nu_{k}\right] \mathrm{E}\left[x_{\ell}^{(i)}(t) \overline{x_{\ell}^{(i)}(u)}\right] \mathrm{d} u \mathrm{~d} t \\
& =\sum_{m, m^{\prime}=-\infty}^{\infty} \mathrm{E}\left[b_{k}[m] b_{k}\left[m^{\prime}\right]\right] \sum_{n, n^{\prime}=\ell N}^{\ell N+N-1} \sum_{j, \iota=0}^{N_{c}-1} \\
& \left.\times \frac{1}{T} \int_{-\infty}^{\infty} \int_{-\infty}^{\infty} e^{\mathrm{j} 2 \pi\left(\nu_{k}-\nu^{(i)}\right.}\right)(u-t) \\
& \times h\left(t-j T_{c}-m T_{0}-\tau_{k}\right) h\left(u-j T_{c}-m^{\prime} T_{0}-\tau_{k}\right) \\
& \times h\left(t-\iota T_{c}-n T_{0}-\tau^{(i)}\right) h\left(u-\iota T_{c}-n^{\prime} T_{0}-\tau^{(i)}\right) \mathrm{d} u \mathrm{~d} t,
\end{aligned}
$$

where we used expressions like $\sum_{n, n^{\prime}=\ell N}^{\ell N+N-1}$ to indicate a double sum $\sum_{n=\ell N}^{\ell N+N-1} \sum_{n^{\prime}=\ell N}^{\ell N+N-1}$ whenever the two sets of 
summation indices are identical. As a consequence of the Plancherel theorem [40], for any $t_{0}, t_{1} \in \mathbb{R}$ and $f_{0} \in \mathbb{R}$

$$
\begin{aligned}
& \int_{-\infty}^{\infty} e^{\mathrm{j} 2 \pi f_{0} t} h\left(t-t_{0}\right) h\left(t-t_{1}\right) \mathrm{d} t \\
& =e^{\mathrm{j} 2 \pi f_{0} t_{0}} \int_{-\infty}^{\infty} \overline{H(f)} H\left(f-f_{0}\right) e^{\mathrm{j} 2 \pi f\left(t_{1}-t_{0}\right)} \mathrm{d} f \\
& \approx e^{\mathrm{j} 2 \pi f_{0} t_{0}} \int_{-\infty}^{\infty}|H(f)|^{2} e^{\mathrm{j} 2 \pi f\left(t_{1}-t_{0}\right)} \mathrm{d} f
\end{aligned}
$$

where the approximation $H\left(f-f_{0}\right) \approx H(f)$ is reasonably accurate if $\left|f_{0}\right| \ll 1 / T_{c}$, which is the case for Doppler frequencies $f_{0}$ on the order of $\mathrm{kHz}$ and chip rates $1 / T_{c}$ on the order of $\mathrm{MHz}$, as is typical in the context of GNSS. Applying (55) to (54), we obtain

$$
\begin{aligned}
& \operatorname{Var}\left[X_{k}^{(i)}[\ell] \mid \tau_{k}, \nu_{k}\right] \\
& =\frac{1}{T} \sum_{m, m^{\prime}=-\infty}^{\infty} \mathrm{E}\left[b_{k}[m] b_{k}\left[m^{\prime}\right]\right] \sum_{n, n^{\prime}=\ell N}^{\ell N+N-1} \sum_{j, \iota=0}^{N_{c}-1} \\
& \times e^{\mathrm{j} 2 \pi\left(\nu^{(i)}-\nu_{k}\right)\left(j T_{c}+m T_{0}+\tau_{k}\right)} e^{\mathrm{j} 2 \pi\left(\nu_{k}-\nu^{(i)}\right)\left(j T_{c}+m^{\prime} T_{0}+\tau_{k}\right)} \\
& \times \int_{-\infty}^{\infty}|H(f)|^{2} e^{\mathrm{j} 2 \pi f\left((\iota-j) T_{c}+(n-m) T_{0}+\tau^{(i)}-\tau_{k}\right)} \mathrm{d} f \\
& \times \int_{-\infty}^{\infty}|H(s)|^{2} e^{\mathrm{j} 2 \pi s\left((\iota-j) T_{c}+\left(n^{\prime}-m^{\prime}\right) T_{0}+\tau^{(i)}-\tau_{k}\right)} \mathrm{d} s
\end{aligned}
$$

The symbol autocorrelation from Fig. 1 can be written as a weighted sum of delayed unit impulses

$$
\mathrm{E}\left[b_{k}[m] b_{k}\left[m^{\prime}\right]\right]=\sum_{\mu=1-M}^{M-1}\left(1-\frac{|\mu|}{M}\right) \delta\left[m-m^{\prime}-\mu\right]
$$

Thus we can perform the summation over $m^{\prime}$ and have

$$
\begin{aligned}
& \operatorname{Var}\left[X_{k}^{(i)}[\ell] \mid \tau_{k}, \nu_{k}\right] \\
& =\frac{1}{T} \sum_{\mu=1-M}^{M-1}\left(1-\frac{|\mu|}{M}\right) e^{\mathrm{j} 2 \pi\left(\nu^{(i)}-\nu_{k}\right) \mu T_{0}} \sum_{n, n^{\prime}=\ell N}^{\ell N+N-1} \sum_{j, \iota=0}^{N_{c}-1} \\
& \times \int_{-\infty}^{\infty}|H(f)|^{2} e^{\mathrm{j} 2 \pi f\left((\iota-j) T_{c}+n T_{0}+\tau^{(i)}-\tau_{k}\right)} \\
& \times \int_{-\infty}^{\infty}|H(s)|^{2} e^{\mathrm{j} 2 \pi s\left((\iota-j) T_{c}+\left(n^{\prime}+\mu\right) T_{0}+\tau^{(i)}-\tau_{k}\right)} \\
& \times \sum_{m=-\infty}^{\infty} e^{-\mathrm{j} 2 \pi(f+s) m T_{0}} \mathrm{~d} f \mathrm{~d} s
\end{aligned}
$$

Applying the Poisson summation formula [40]

$$
\sum_{m=-\infty}^{\infty} e^{-\mathrm{j} 2 \pi(f+s) m T_{0}}=\frac{1}{T_{0}} \sum_{m=-\infty}^{\infty} \delta\left(f+s-\frac{m}{T_{0}}\right),
$$

we can perform the integration over $s$

$$
\begin{aligned}
& \operatorname{Var}\left[X_{k}^{(i)}[\ell] \mid \tau_{k}, \nu_{k}\right] \\
& =\frac{1}{T T_{0}} \sum_{\mu=1-M}^{M-1}\left(1-\frac{|\mu|}{M}\right) e^{\mathrm{j} 2 \pi\left(\nu^{(i)}-\nu_{k}\right) \mu T_{0}} \sum_{n, n^{\prime}=\ell N}^{\ell N+N-1} \sum_{j, \iota=0}^{N_{c}-1} \\
& \times \sum_{m=-\infty}^{\infty} e^{\mathrm{j} 2 \pi \frac{m}{T_{0}}\left((\iota-j) T_{c}+\tau^{(i)}-\tau_{k}\right)} \\
& \times \int_{-\infty}^{\infty}|H(f)|^{2}\left|H\left(\frac{m}{T_{0}}-f\right)\right|^{2} e^{\mathrm{j} 2 \pi f\left(n-n^{\prime}-\mu\right) T_{0}} \mathrm{~d} f \\
& =\sum_{\mu=1-M n=1-N}^{M-1} \sum_{n-1}\left(1-\frac{|\mu|}{M}\right)\left(1-\frac{|n|}{N}\right) e^{\mathrm{j} 2 \pi\left(\nu^{(i)}-\nu_{k}\right) \mu T_{0}} \\
& \times \sum_{m=-\infty}^{\infty} e^{\mathrm{j} 2 \pi \frac{m}{T_{0}}\left(\tau^{(i)}-\tau_{k}\right)} \frac{1}{N_{c}} \sum_{j=1-N_{c}}^{N_{c}-1}\left(1-\frac{|j|}{N_{c}}\right) e^{\mathrm{j} \frac{2 \pi m}{N_{c}} j} \\
& \times \frac{1}{T_{c}^{2}} \int_{-\infty}^{\infty}|H(f)|^{2}\left|H\left(\frac{m}{T_{0}}-f\right)\right|^{2} e^{\mathrm{j} 2 \pi f(n-\mu) T_{0}} \mathrm{~d} f, \quad(60)
\end{aligned}
$$

where we replaced the double summations over $n, n^{\prime}$ and $j, \iota$ by simple summations using the identity

$$
\sum_{n=n_{0}}^{n_{0}+N} \sum_{n^{\prime}=n_{0}}^{n_{0}+N} g\left(n-n^{\prime}\right)=N \sum_{n=1-N}^{N-1}\left(1-\frac{|n|}{N}\right) g(n)
$$

for $n_{0}, N \in \mathbb{Z}$ and any locally summable function $g: \mathbb{R} \rightarrow \mathbb{C}$. Furthermore, note that

$$
\sum_{j=1-N_{c}}^{N_{c}-1}\left(1-\frac{|j|}{N_{c}}\right) e^{-\mathrm{j} \frac{2 \pi m}{N_{c}} j}=N_{c} \sum_{j=-\infty}^{\infty} \delta\left[m-j N_{c}\right],
$$

which we use to perform the summation over $m$ in (60)

$$
\begin{aligned}
& \operatorname{Var}\left[X_{k}^{(i)}[\ell] \mid \tau_{k}, \nu_{k}\right] \\
& =\sum_{\mu=1-M}^{M-1} \sum_{n=1-N}^{N-1}\left(1-\frac{|\mu|}{M}\right)\left(1-\frac{|n|}{N}\right) e^{\mathrm{j} 2 \pi\left(\nu^{(i)}-\nu_{k}\right) \mu T_{0}} \\
& \times \sum_{j=-\infty}^{\infty} e^{\mathrm{j} 2 \pi \frac{j}{T_{c}}\left(\tau^{(i)}-\tau_{k}\right)} \\
& \times \frac{1}{T_{c}^{2}} \int_{-\infty}^{\infty}|H(f)|^{2}\left|H\left(\frac{j}{T_{c}}-f\right)\right|^{2} e^{\mathrm{j} 2 \pi f(n-\mu) T_{0}} \mathrm{~d} f .
\end{aligned}
$$

The integral in (63) vanishes for terms $\mu \neq n$ if either REC, $\mathrm{BOC}$, or RRC pulse with zero roll-off are used as pulse shape, as the following calculation shows. Taking into account the even symmetry of $|H(f)|^{2}$, we can use the identity (55) once again to show that

$$
\begin{aligned}
& \int_{-\infty}^{\infty}|H(f)|^{2}\left|H\left(\frac{j}{T_{c}}-f\right)\right|^{2} e^{\mathrm{j} 2 \pi f(n-\mu) T_{0}} \mathrm{~d} f \\
& =\int_{-\infty}^{\infty} e^{\mathrm{j} 2 \pi \frac{j}{T_{c}} t} \rho_{h}\left(t-\mu T_{0}\right) \rho_{h}\left(t-n T_{0}\right) \mathrm{d} t \\
& \approx \delta[n-\mu] \int_{-\infty}^{\infty} e^{\mathrm{j} 2 \pi \frac{j}{T_{c}} t} \rho_{h}^{2}\left(t-n T_{0}\right) \mathrm{d} t \\
& =\delta[n-\mu] \int_{-\infty}^{\infty}|H(f)|^{2}\left|H\left(\frac{j}{T_{c}}-f\right)\right|^{2} \mathrm{~d} f .
\end{aligned}
$$


The approximation in (64) is exact for all time-limited pulse shapes such as REC or BOC, for which the product $\rho_{h}\left(t-\mu T_{0}\right) \rho_{h}\left(t-n T_{0}\right)$ can be nonzero only if $\mu=n$. The approximation is also exact for the RRC pulse with zero rolloff (also known as flat spectrum pulse), because then all terms $j \neq 0$ in (64) vanish entirely and $\rho_{h}(t)$ is itself a Nyquist pulse. Numerical computations of the above terms for $\mu \neq n$ led to negligible values for many other pulses as well, which is not surprising as $\rho_{h}(t)$ usually decays over time with $1 / t$ or faster.

Substituting (64) into (63) and performing the summation over $\mu$, we have finally

$$
\begin{aligned}
& \operatorname{Var}\left[X_{k}^{(i)}[\ell] \mid \tau_{k}, \nu_{k}\right] \\
& =\sum_{n=1-N}^{N-1}\left(1-\frac{|n|}{M}\right)\left(1-\frac{|n|}{N}\right) e^{\mathrm{j} 2 \pi\left(\nu^{(i)}-\nu_{k}\right) n T_{0}} \\
& \times \sum_{j=-\infty}^{\infty} e^{\mathrm{j} 2 \pi \frac{j}{T_{c}}\left(\tau^{(i)}-\tau_{k}\right)} \\
& \times \frac{1}{T_{c}^{2}} \int_{-\infty}^{\infty}|H(f)|^{2}\left|H\left(\frac{j}{T_{c}}-f\right)\right|^{2} \mathrm{~d} f,
\end{aligned}
$$

which is equivalent to the desired results for the SSC-D (22)(27). The desired results for the SSC (16)-(17) can now easily be obtained by removing the conditioning on $\tau_{k}, \nu_{k}$. Upon taking the expectation, all summands $n \neq 0$ and $j \neq 0$ vanish

$$
\mathrm{E}\left[\operatorname{Var}\left[X_{k}^{(i)}[\ell] \mid \tau_{k}, \nu_{k}\right]\right]=\frac{1}{T_{c}^{2}} \int_{-\infty}^{\infty}|H(f)|^{4} \mathrm{~d} f
$$

\section{REFERENCES}

[1] E. D. Kaplan and C. Hegarty, Understanding GPS: principles and applications. Boston: Artech House, 2006.

[2] U. Cheng, W. Hurd, and J. Statman, "Spread-spectrum code acquisition in the presence of Doppler shift and data modulation," IEEE Transactions on Communications, vol. 38, no. 2, pp. 241-250, Feb. 1990.

[3] K. Chen, G. Tan, J. Cao, M. Lu, and X. Fan, "Modeling and Improving the Energy Performance of GPS Receivers for Location Services," IEEE Sensors Journal, vol. 20, no. 8, pp. 4512-4523, Apr. 2020.

[4] F. S. T. Van Diggelen, A-GPS: Assisted GPS, GNSS, and SBAS. Artech House, 2009.

[5] F. van Diggelen, "Assisted GNSS," in Position, Navigation, and Timing Technologies in the 21st Century. John Wiley \& Sons, Ltd, 2020, pp. 419-444.

[6] D. Borio, L. Camoriano, and L. Lo Presti, "Impact of GPS acquisition strategy on decision probabilities," IEEE Transactions on Aerospace and Electronic Systems, vol. 44, no. 3, pp. 996-1011, Jul. 2008.

[7] C. O'Driscoll, "Performance analysis of the parallel acquisition of weak GPS signals," Ph.D. dissertation, University College Cork, 2007.

[8] "Power-efficient positioning for the Internet of Things: merging GNSS with low power connectivity solutions: white paper," European GNSS Agency, Publications Office of the EU, Tech. Rep., 2020.

[9] G. Corazza, "On the MAX/TC criterion for code acquisition and its application to DS-SSMA systems," IEEE Transactions on Communications, vol. 44, no. 9, pp. 1173-1182, Sep. 1996.

[10] A. Coenen and D. van Nee, "Novel fast GPS/GLONASS codeacquisition technique using low update rate FFT," Electronics Letters, vol. 28, no. 9, p. 863, 1992.

[11] F. Van Diggelen, "Who's Your Daddy? Why GPS Will Continue to Dominate Consumer GNSS," Inside GNSS, vol. 9, no. 2, Mar. 2014.

[12] S. Wallner, "Galileo System and Signal Evolution," Int. Techn. Symposium on Navigation and Timing, Toulouse, France, Nov. 2017.

[13] M. Paonni and M. Bavaro, "On the Design of a GNSS Acquisition Aiding Signal," in Proceedings of the 26th International Technical Meeting of the Satellite Division of The Institute of Navigation (ION GNSS+ 2013), Nashville, Tennessee, Sep. 2013, pp. 1445-1456.
[14] M. Paonni, M. Anghileri, D. Fontanella, and B. Eissfeller, "Quasi-Pilot Signals: Improving Sensitivity and TTFF without Compromises," in Proceedings of the 24th International Technical Meeting of the Satellite Division of The Institute of Navigation (ION GNSS 2011), Portland, Oregon, Sep. 2011, pp. 1254-1263.

[15] S. Wallner, J. A. Garcia Molina, G. L. Risueno, J. Hahn, F. Jean Jeacques, F. Soualle, T. Schmitt, G. da Broi, M. Ouedraogo, T. Woerz, G. de Pasquale, C. Vazquez, and M. Paonni, "Novel Concepts on GNSS Signal Design serving Emerging GNSS User Categories: Quasi-Pilot Signal," in European Navigation Conference (ENC) 2020. Dresden, Germany: DGON, Nov. 2020.

[16] T. A. Stansell, J. W. Betz, F. van Diggelen, and S. Kogure, "Proposed Evolution of the C/A Signal," in Proceedings of the 28th International Technical Meeting of the Satellite Division of The Institute of Navigation (ION GNSS+ 2015), Tampa, Florida, Sep. 2015, pp. 1807-1825.

[17] S. Qaisar and A. Dempster, "Cross-correlation performance assessment of global positioning system (GPS) L1 and L2 civil codes for signal acquisition," IET Radar, Sonar \& Navigation, vol. 5, no. 3, p. 195, 2011.

[18] J. W. Betz and D. B. Goldstein, "Candidate designs for an additional civil signal in GPS spectral bands," in Proceedings of the 2002 National Technical Meeting of The Institute of Navigation, San Diego, California, Jan. 2002, pp. 622-631.

[19] J. W. Betz and K. R. Kolodziejski, "Generalized theory of code tracking with an early-late discriminator part i: Lower bound and coherent processing," IEEE Transactions on Aerospace and Electronic Systems, vol. 45, no. 4, pp. 1538-1556, 2009.

[20] C. O'Driscoll and J. Fortuny-Guasch, "On the Determination of C/A Code Self-Interference with Application to RFC Analysis and Pseudolite Systems," in Proceedings of the 25th International Technical Meeting of The Satellite Division of the Institute of Navigation (ION GNSS 2012), Nashville, TN, Sep. 2012, pp. 3620-3631.

[21] A. Van Dierendonck, S. Kalyanaraman, C. J. Hegarty, and K. Shallberg, "A more accurate Evaluation of GPS C/A Code Self-Interference Considering Critical Satellites," in Proceedings of the 19th International Technical Meeting of the The Institute of Navigation, Monterey, California, Jan. 2017, pp. 671-680.

[22] C. Enneking, F. Antreich, L. Krieger, and A. L. F. de Almeida, "Gaussian Approximations for Intra- and Intersystem Interference in RNSS," IEEE Communications Letters, vol. 23, no. 7, pp. 1198-1201, Jul. 2019, conference Name: IEEE Communications Letters.

[23] C. Enneking, F. Antreich, M. M. Appel, and A. L. de Almeida, "Pure Pilot Signals: How Short can we Choose GNSS Spreading Codes?" in 2019 International Technical Meeting of The Institute of Navigation, Reston, Virginia, Feb. 2019, pp. 925-935.

[24] C. J. Hegarty, "A simple model for GPS C/A-code self-interference," NAVIGATION, vol. 67, no. 2, pp. 319-331, Jun. 2020.

[25] European GNSS (Galileo) Open Service: Signal-in-Space Interface Control Document, European Union, OS SIS ICD Issue 1.3, Interface Specification, 2016

[26] D. Borio, "A Statistical Theory for GNSS Signal Acquisition," Ph.D. dissertation, Politecnico di Torino, 2008.

[27] J. H. Cho and J. S. Lehnert, "An optimal signal design for bandlimited asynchronous DS-CDMA communications," IEEE Transactions on Information Theory, vol. 48, no. 5, pp. 1172-1185, May 2002.

[28] G. Zang and C. Ling, "Performance evaluation for band-limited DSCDMA systems based on simplified improved Gaussian approximation," IEEE Transactions on Communications, vol. 51, no. 7, pp. 1204-1213, Jul. 2003.

[29] C. Enneking, F. Antreich, and A. L. F. de Almeida, "Early-Late Discriminator Performance of CDMA: Limitations of the Spectral Separation Coefficient," in NAVITEC 2018 Signal Workshop, Noordwijk, The Netherlands, Dec. 2018.

[30] J. W. Betz, "Effect of Narrowband Interference on GPS Code Tracking Accuracy," in Proceedings of the 2000 National Technical Meeting of The Institute of Navigation, Anaheim, CA, Jan. 2000, pp. 16-27.

[31] _ , "Effect of Partial-Band Interference on Receiver Estimation of C/NO: Theory," in Proceedings of the 2001 National Technical Meeting of The Institute of Navigation, Long Beach, CA, Jan. 2001, pp. 817-828.

[32] European GNSS (Galileo) Initial Services: Open Service, European Union, OS SDD Issue 1.0, Service Definition Document, 2016.

[33] "Assessment of Radio Frequency Interference Relevant to the GNSS L1 Frequency Band," RTCA, Inc., DO-235, Mar. 2008.

[34] A. Papoulis, Probability, Random Variables, and Stochastic Processes, ser. Communications and signal processing. McGraw-Hill, 1991.

[35] M. Foucras, O. Julien, C. Macabiau, B. Ekambi, and F. Bacard, "Probability of detection for gnss signals with sign transitions," IEEE 
Transactions on Aerospace and Electronic Systems, vol. 52, no. 3, pp. 1296-1308, 2016.

[36] J. Marcum, "A statistical theory of target detection by pulsed radar," IRE Transactions on Information Theory, vol. 6, no. 2, pp. 59-267, 1960.

[37] "NAVSTAR GPS Space Segment/Navigation User Interfaces," IS-GPS200, Rev. L, GPS Enterprise, El Segundo, CA, May 2020.

[38] N. Ziedan, GNSS receivers for weak signals. Boston, Lodon: Artech House, 2006.

[39] C. Enneking, F. Antreich, and A. L. F. de Almeida, "GNSS Acquisition Performance of Short Spreading Codes," in 33rd International Technical Meeting of the Satellite Division of The Institute of Navigation (ION GNSS+ 2020), Oct. 2020, pp. 1238-1260.

[40] K. Yosida, Functional Analysis, ser. Classics in Mathematics. SpringerVerlag Berlin Heidelberg, 1995, no. 6th ed.

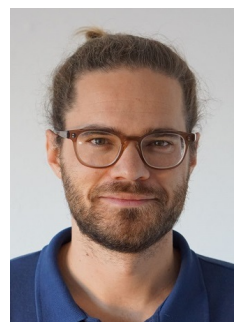

Christoph Enneking received the master degree (MSc.) in electrical engineering from the Munich University of Technology (TUM), Germany, in 2014. He received the doctor degree (Ph.D.) in teleinformatics engineering from the Federal University of Ceará (UFC), Brazil, in 2020. In 2014, he joined the Institute of Communications and Navigation of the German Aerospace Center (DLR), WesslingOberpfaffenhofen. His research interests include GNSS signal design, estimation theory, and GNSS intra- and intersystem interference.

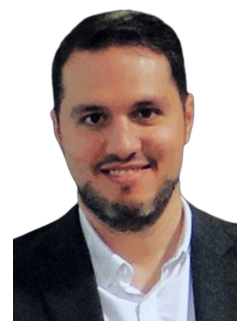

André L. F. de Almeida (M'08-SM'13) is an Associate Professor with the Department of Teleinformatics Engineering at the Federal University of Ceará. He served as an Associate Editor for the IEEE Transactions on Signal Processing (2012-2016). He currently serves as a Senior Area Editor for the IEEE Signal Processing Letters and an Associate Editor of the IEEE Transactions on Vehicular Technology. Dr. Almeida is an elected member of the Sensor Array and Multichannel Technical Committee of the IEEE Signal Processing Society and an elected member of the EURASIP Signal Processing for Multi-Sensor Systems Technical Area Committee. He has over 200 refereed published articles in journals and conferences. He was the general co-chair of the IEEE CAMSAP'2017 workshop and served as Symposia Technical Co-Chair at IEEE GlobalSIP 2018 and 2019. He was a Technical Co-Chair of IEEE SAM 2020 workshop, Hangzhou, China $\mathrm{He}$ also serves as the general co-chair of the IEEE CAMSAP'2023, Costa Rica. He is a research fellow of the CNPq (the Brazilian National Council for Scientific and Technological Development) and an Affiliate Member of the Brazilian Academy of Sciences. His research interests include multilinear algebra and tensor decompositions with applications to communications and signal processing.

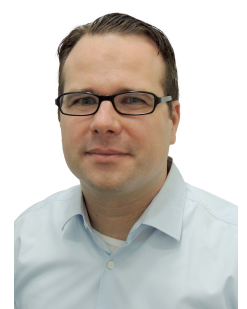

Felix Antreich (M'06-SM'17) received the Diploma degree in electrical engineering from the Technical University of Munich (TUM), Munich, Germany, in 2003. In 2011 he also received the Doktor-Ingenieur (Ph.D.) degree from the TUM. From 2003 to 2016, he was an Associate Researcher with the Department of Navigation, Institute of Communications and Navigation of the German Aerospace Center (DLR), Wessling, Germany. From 2016 to 2018 he was a Visiting Professor in the Department of Teleinformatics Engineering (DETI) at the Federal University of Ceará (UFC) in Fortaleza, Brazil. Since July 2018 he is a Professor with the Department of Telecommunications in the Division of Electronics Engineering of the Aeronautics Institute of Technology (ITA) in São José dos Campos, Brazil. His research interests include sensor array signal processing for global navigation satellite systems (GNSS) and wireless communications, estimation theory, wireless sensor networks, positioning, localization, and signal design for synchronization. 